GEOMETRY OF LAGRANGIAN GRASSMANNIANS

AND NONLINEAR PDES

BANACH CENTER PUBLICATIONS, VOLUME 117

INSTITUTE OF MATHEMATICS

POLISH ACADEMY OF SCIENCES

WARSZAWA 2019

\title{
PROJECTIVE DUALITY AND NON-DEGENERATED SYMPLECTIC MONGE-AMPĖRE EQUATIONS
}

\author{
FRANCESCO RUSSO \\ Dipartimento di Matematica e Informatica, Università degli Studi di Catania \\ Viale A. Doria 6, 95125 Catania, Italy \\ E-mail: frusso@dmi.unict.it
}

\begin{abstract}
The aim of these notes is to introduce the basic notions of projective duality and of secant varieties in order to provide a firm background to the geometrical counterpart of the main results by Doubrov and Ferapontov [J. Geom. Phys. 60 (2010), 1604-1616] and Ferapontov, Hadjikos and Khusnutdinova [Int. Math. Res. Not. IMRN 2010, 496-535] on the integrability of non-degenerate symplectic Monge-Ampère equations
\end{abstract}

\section{Contents}

1. Introduction . . . . . . . . . . . . . . . . . . . . . . 114

2. Dual varieties . . . . . . . . . . . . . . . . . . . . . . . . 117

3. Some examples revisited . . . . . . . . . . . . . . . . . . . . . . . . 119

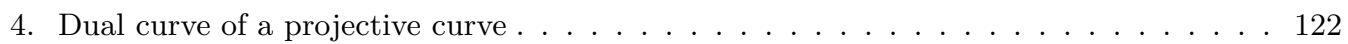

$5 . \quad L G(3,6)$ as twisted cubic over an algebra $\ldots \ldots \ldots \ldots \ldots \ldots . \ldots \ldots$

$6 . \quad$ Tangent cones and tangent spaces of an algebraic variety. . . . . . . . . . . . . . . . 129

7. Join of varieties . . . . . . . . . . . . . . . . . . . . . . . . . 133

8. Terracini's Lemma and its first applications . . . . . . . . . . . . . . . . . . . 135

9. Terracini's Lemma and $L G(4,8)$. . . . . . . . . . . . . . . . . . . . . . . . . . . . 140

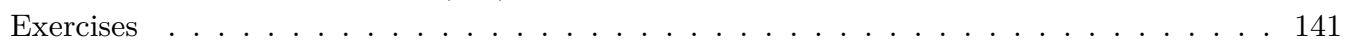

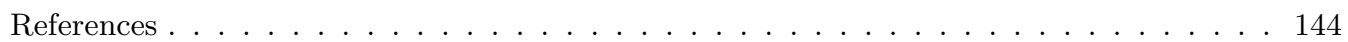

2010 Mathematics Subject Classification: Primary 14N05, 14M17; Secondary 32W20.

Key words and phrases: Lagrangian Grassmannian, dual variety, symplectic Monge-Ampère equations.

Partially supported by PRIN Grant Geometria delle varietà algebriche and by the Research Project FIR 2014 Aspetti geometrici e algebrici della Weak e Strong Lefschetz Property of the UNICT. The author is a member of the GNSAGA group of INDAM.

The paper is in final form and no version of it will be published elsewhere. 
Foreword. These notes contain an expanded version of a series of lectures presented in the Workshop on the Geometry of Lagrangian Grassmannians and nonlinear PDEs, Warsaw 5-9/9/2016.

They are essentially based on the material contained in [10], in [8] and on some unpublished notes of the author.

It is a pleasure to thank the Organizers of the Workshop for inviting me and for giving to me the opportunity of lecturing on these topics. In particular, I wish to express my gratitude to Giovanni and Gianni with whom I discussed several times on these or on related subjects and from whom I learned some beautiful applications of the geometry of projective varieties to nonlinear PDEs. They also helped me in the typesetting of these notes and without their contribution I would have not been able to finalize this project in due time.

1. Introduction. The aim of the mini-course is to provide a complete algebro-geometric background of some notions appearing in a series of papers by Ferapontov and his collaborators about the integrability of non-degenerated symplectic Monge-Ampère equations in low dimensions $(n=3,4)$.

Let us recall that, if

$$
U=\left[u_{i j}\right]
$$

is the Hessian matrix of a function

$$
u=u\left(x_{1}, \ldots, x_{n}\right),
$$

then a symplectic Monge-Ampère equation is a linear combination of all minors of $U$,

$$
a+b_{1} u_{1,1}+\ldots+b_{n(n+1) / 2} u_{n, n}+c_{1}\left(u_{1,1} u_{1,2}-u_{1,2}^{2}\right)+\ldots+\beta \operatorname{det}(U)=0
$$

Taking

$$
A=\left[\begin{array}{ccc}
y_{1,1} & y_{1,2} & \vdots \\
y_{1,2} & y_{2,2} & \vdots \\
\cdots & \cdots & y_{n, n}
\end{array}\right]=A^{t}
$$

a generic symmetric matrix of order $n$, we can construct a projective variety of dimension $\frac{n(n+1)}{2}$ with an embedding in a projective space $\mathbb{P}^{N(n)}$ with

$$
N(n)=\left(\begin{array}{c}
2 n \\
n
\end{array}\right)-\left(\begin{array}{c}
2 n \\
n-2
\end{array}\right)-1
$$

by taking

$$
X:=\overline{\left\{\left(1: A: 2 \times 2 \text { minors of } A: 3 \times 3 \text { minors of } A: \ldots: A^{\sharp}: \operatorname{det}(A)\right)\right\}} \subset \mathbb{P}^{N(n)} .
$$

The matrix $A^{\sharp}$ is defined by the formula

$$
A^{\sharp} \cdot A=\operatorname{det}(A) \cdot I_{n \times n} .
$$

We call $X:=\operatorname{LG}(n, 2 n) \subset \mathbb{P}^{N(n)}$ the Lagrangian Grassmannian, which a smooth homogeneous projective variety of dimension

$$
\operatorname{dim} \operatorname{LG}(n, 2 n)=\frac{n(n+1)}{2} .
$$


EXAMPLE 1.1. If $n=2$, then

and

$$
\operatorname{dim} \operatorname{LG}(2,4)=\frac{2 \cdot 3}{2}=3
$$

$$
N(2)=\left(\begin{array}{l}
4 \\
2
\end{array}\right)-\left(\begin{array}{l}
4 \\
0
\end{array}\right)-1=6-2=4 \text {. }
$$

With

$$
\mathbb{P}^{4}=\left\{\left[y_{0}: y_{1}: y_{2}: y_{3}: y_{4}\right]\right\}
$$

and

$$
A=\left[\begin{array}{ll}
a & b \\
c & d
\end{array}\right]
$$

the algebraic subset $\operatorname{LG}(2,4) \subset \mathbb{P}^{4}$ is parametrically given by

$$
y_{0}=1, y_{1}=a, y_{2}=b, y_{3}=c, y_{4}=a c-b^{2},
$$

and, hence, implicitly by

$$
y_{1} y_{3}-y_{2}^{2}-y_{0} y_{4}=0,
$$

which is the equation of a smooth quadric hypersurface.

EXAMPLE 1.2. For $n=3$,

$$
\mathrm{LG}(3,6)=\overline{\left\{\left(1: A: A^{\sharp}: \operatorname{det}(A)\right)\right\}} \subset \mathbb{P}^{N(3)}=\mathbb{P}^{13},
$$

where

$$
A=A^{t}=\left(\begin{array}{lll}
y_{1} & y_{2} & y_{3} \\
y_{2} & y_{4} & y_{5} \\
y_{3} & y_{5} & y_{6}
\end{array}\right)
$$

and the $y_{6+j}$, with $j=1,2, \ldots, 6$ are the cofactors of $A$, and $y_{13}$ its determinant.

EXAmple 1.3. For $n=4$, one has

$$
\mathrm{LG}(4,8) \subset \mathbb{P}^{41} \text { and } \operatorname{dim} \operatorname{LG}(4,8)=10 .
$$

From this point of view, symplectic Monge-Ampère equations of dimension $n$ correspond to hyperplane sections of

$$
\mathrm{LG}(n, 2 n) \subset \mathbb{P}^{N(n)} .
$$

Let us recall some results of Ferapontov and his collaborators to motivate the study of projective duality.

From now on we shall identify symplectic Monge-Ampère equations of dimension $n$ and hyperplane sections. Let

$$
\left(a_{0}: a_{1}: \ldots: a_{N(n)}\right)
$$

be coordinates on $\left(\mathbb{P}^{N(n)}\right)^{*}$ and let

$$
H: \sum_{j=0}^{N(n)} \widetilde{a}_{j} x_{j}=0
$$

denote a hyperplane in $\mathbb{P}^{N(n)}$ with coordinates $\left(x_{0}: \ldots: x_{N(n)}\right)$.

With this notation we have the following results, see also Example 4.9 . 
Theorem 1.4 (Doubrov-Ferapontov, [6]). A non-degenerate symplectic Monge-Ampère equation of dimension $n \geq 2$ is linearizable by a transformation of $\operatorname{Sp}(2 n)$ if and only if the corresponding hyperplane $H \subset \mathbb{P}^{N(n)}$ is such that $H \supset T_{x}^{n-2} \mathrm{LG}(n, 2 n)$, where $T_{x}^{n-2} \mathrm{LG}(n, 2 n)$ is the $(n-2)$-osculating space to $\mathrm{LG}(n, 2 n) \subset \mathbb{P}^{N(n)}$ at some point $x$.

Theorem 1.5 (Ferapontov et al., see [7]). A non-degenerate symplectic Monge-Ampère equation of dimension 3 is integrable if and only if the corresponding hyperplane $H \subset \mathbb{P}^{13}$ is such that $H \supset T_{x} \mathrm{LG}(3,6)$, that is, if $H$ is tangent to $\mathrm{LG}(3,6)$ at some point $x$ (equivalently, $H \cap X$ is singular at (some point) $x$ ). In particular, such equations are integrable if and only if they are linearizable.

Therefore (1) is integrable if and only if the coordinates of the corresponding $H$ as in (2), $\left(\widetilde{a}_{0}: \ldots: \widetilde{a}_{13}\right)$, satisfy an equation of degree 4 in the variables $\left(a_{0}: \ldots: a_{13}\right)$, which is the equation of the dual variety of $\mathrm{LG}(3,6)$.

TheOrem 1.6 (Doubrov-Ferapontov, [6]). A non-degenerate symplectic Monge-Ampère equation of dimension 4 is integrable if and only if $H \cap X$ is singular along a 4-dimensional variety $Z^{4}$ which intersect all $\mathrm{LG}(3,6) \subset \mathrm{LG}(4,8)$.

These results should have motivated sufficiently the audience of the workshop (and/or the readers) to undertake a systematic study of singular (or tangent) hyperplane sections of an arbitrary irreducible algebraic variety $X \subset \mathbb{P}^{N}$ (over $\mathbb{C}$ ).

If $H \in\left(\mathbb{P}^{N}\right)^{*}=\mathbb{P}\left(V^{*}\right)$ is a hyperplane, with $\mathbb{P}^{N}=\mathbb{P}(V)$, then

$$
X^{*}:=\overline{\left\{[H] \in\left(\mathbb{P}^{N}\right)^{*} \mid H \cap X \text { is singular outside } \operatorname{Sing}(X)\right\}} \subseteq\left(\mathbb{P}^{N}\right)^{*}
$$

is the dual variety of $X$. Before presenting the formal theory let us describe some examples and their connections with the previous results.

EXAMPLE 1.7. $\operatorname{LG}(3,6)^{*} \subset\left(\mathbb{P}^{13}\right)^{*}$ is a hypersurface of degree 4 : how to calculate it? does it have a different geometrical description?

ExAmPLE 1.8. $\operatorname{LG}(4,8)^{*} \supset\{[H] \mid H \cap X$ is integrable $\}$ : what is it? how to describe geometrically these $H$ 's?

A guiding example to understand the peculiarities of the geometry of LG(3,6) and of $\mathrm{LG}(4,8)$ is the case of the twisted cubic in $\mathbb{P}^{3}$, respectively the quartic rational normal curve $\nu_{4}\left(\mathbb{P}^{1}\right) \subset \mathbb{P}^{4}$.

ExAmple 1.9. Let $X=v_{3}\left(\mathbb{P}^{1}\right) \subset \mathbb{P}^{3}$ be the twisted cubic. Then

$$
v_{3}\left(\mathbb{P}^{1}\right)=\overline{\left\{\left(1: t: t^{2}: t^{3}\right) \mid t \in \mathbb{C}\right\}} \subset \mathbb{P}^{3}
$$

reminds the parametrization of $\operatorname{LG}(3,6)$.

Let

$$
\boldsymbol{a}=\left(a_{0}: a_{1}: a_{2}: a_{3}\right)
$$

and

$$
H \cap X=\left\{t \in \mathbb{C} \mid a_{0}+a_{1} t+a_{2} t^{2}+a_{3} t^{3}=0\right\} .
$$

Thus, $H \cap X$ is singular (or equivalently $H$ is tangent to $X$ ) if and only if (3) has multiple roots, i.e. if and only if its coordinates satisfy

$$
\Delta\left(a_{0}, a_{1}, a_{2}, a_{3}\right)=a_{1}^{2} a_{2}^{2}-4 a_{3} a_{1}^{3}-4 a_{2}^{3} a_{0}-27 a_{3}^{2} a_{2}^{2}+18 a_{0} a_{1} a_{2} a_{3}=0 .
$$


The above discriminant of the equation of third order has degree four so that

$$
\left(\nu_{3}\left(\mathbb{P}^{1}\right)\right)^{*}=V(\Delta) \subset\left(\mathbb{P}^{3}\right)^{*}
$$

is a hypersurface of degree 4 .

2. Dual varieties and contact loci of general tangent linear spaces. Let $X \subset \mathbb{P}^{N}$ be a projective, irreducible non-degenerate variety of dimension $n=\operatorname{dim}(X)$. Let $X_{\text {reg }}=$ $X \backslash \operatorname{Sing}(X)$ be the locus of non-singular points of $X$ and let $T_{x} X$ be the projective tangent space to $X$ at $x$. One has

$$
X_{\text {reg }}=\left\{x \in X \mid \operatorname{dim}\left(T_{x} X\right)=n\right\} .
$$

If we take a hyperplane section of $X, Y=X \cap H$, where $H=\mathbb{P}^{N-1}$ is an arbitrary hyperplane, then for every $y \in Y$ we get

$$
T_{y} Y=T_{y} X \cap H .
$$

Since $Y$ is a pure dimensional scheme of dimension $n-1$, we deduce that

$$
\operatorname{Sing}(Y) \backslash(\operatorname{Sing}(X) \cap H)=\left\{y \in Y \backslash(\operatorname{Sing}(X) \cap Y) \mid T_{y} X \subseteq H\right\},
$$

which is an open subset in the locus of points of $X$ at which $H$ is tangent to $X$.

We introduce some further definitions. Let $x \in X_{\text {reg }} \subset \mathbb{P}^{N}$. For any $\ell \in \mathbb{N}$, we denote by

$$
T_{x}^{\ell} X \subset \mathbb{P}^{N}
$$

the $\ell$-th order osculation space of $X$ at $x$. If

$$
\begin{gathered}
\psi:\left(\mathbb{C}^{n}, 0\right) \rightarrow(X, x), \\
u \mapsto \psi(u),
\end{gathered}
$$

is a regular local parametrization of $X$ at $x=\psi(0)$, then $T_{x}^{\ell} X$ can be defined as the projective subspace

$$
\overline{\left\langle\partial^{|\alpha|} \psi(0) / \partial u^{\alpha}\left|\alpha \in \mathbb{N}^{n},\right| \alpha \mid \leq \ell\right\rangle} \subset \mathbb{P}^{N} .
$$

By definition $\operatorname{dim}\left(T_{x}^{\ell} X\right) \leq\left(\begin{array}{c}n+\ell \\ n\end{array}\right)-1$ and in general it is expected that equality holds at general points of $X \subset \mathbb{P}^{N}$ as soon as $N \geq\left(\begin{array}{c}n+\ell \\ n\end{array}\right)-1$. In this case, we shall say that the osculation of order $\ell$ of $X$ at $x$ is regular.

Let us remark that, if $T_{x}^{\ell} X \subsetneq \mathbb{P}^{N}$ and if $H \supseteq T_{x}^{\ell} X$ is a hyperplane, then the hyperplane section $H \cap X$ has a point of multiplicity at least $\ell+1$ at $x$. Conversely, $T_{x}^{\ell} X$ can be also defined as the intersection of the hyperplanes $H \subset \mathbb{P}^{N}$ such that $H \cap X$ has a point of multiplicity at least $\ell+1$ at $x$.

The osculating spaces can also be defined more abstractly as the linear subspaces spanned by the $\ell$-th order infinitesimal neighborhood of $X$ at $x$ and also generalized to the case of arbitrary Cartier divisors on $X$.

Indeed, for every integer $\ell \in \mathbb{N}$, let $\mathscr{P}_{X}^{\ell}(D)$ denote the $\ell$-th principal part bundle (or $\ell$-th jet bundle) of $\mathscr{O}_{X}(D)$. For every linear subspace $V \subseteq H^{0}\left(X, \mathscr{O}_{X}(D)\right.$ ) we have a natural homomorphism of sheaves

$$
\phi^{\ell}: V \otimes \mathscr{O}_{X} \rightarrow \mathscr{P}_{X}^{\ell}(D)
$$


sending a section $s \in V$ to its $\ell$-th jet $\phi_{x}^{\ell}(s)$ evaluated at $x \in X$, that is, $\phi_{x}^{\ell}(s)$ is represented in local coordinates by the Taylor expansion of $s$ at $x$, truncated after the order $\ell$. Taking a smooth point $x \in X \subset \mathbb{P}^{N}=\mathbb{P}(V)$ (Grothendieck's notation) and $\mathscr{O}_{X}(D)=\mathscr{O}_{X}(1)$, it is easily verified that $T_{x}^{\ell} X=\mathbb{P}\left(\operatorname{Im}\left(\phi_{x}^{\ell}\right)\right)$.

Therefore to construct a hyperplane section with a non-empty open set of non-singular points, we need to exhibit a hyperplane $H$ which is not tangent to $X$ at all the points in which it intersects $X_{\text {reg. }}$.

There naturally arises the necessity of patching together all the bad hyperplanes and then showing that there always exists a hyperplane section of $X$, non-singular at least outside $\operatorname{Sing}(X)$. Since hyperplanes can be naturally parametrized by points in the dual projective space $\mathbb{P}^{N^{*}}$, we can define a subvariety of $\mathbb{P}^{N^{*}}$ parametrizing hyperplane sections which are also singular outside $\operatorname{Sing}(X)$. This locus is the so-called dual variety defined above which now we reintroduce it in a more abstract way to estimate its expected dimension.

Definition 2.1 (Conormal variety and dual variety). Let $X \subset \mathbb{P}^{N}$ be as above and let

$$
\mathscr{P}_{X}:=\overline{\left\{(x,[H]) \in X_{\mathrm{reg}} \times \mathbb{P}^{N^{*}} \mid T_{x} X \subseteq H\right\}} \subset X \times \mathbb{P}^{N^{*}}
$$

be the conormal variety of $X$.

Let us consider the projections of $\mathscr{P}_{X}$ onto the factors $X$ and $\mathbb{P}^{N^{*}}$,

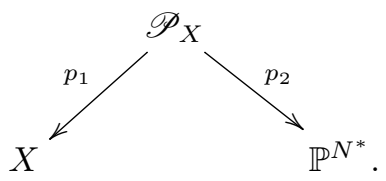

The dual variety to $X$, indicated by $X^{*}$, is the scheme-theoretic image of $\mathscr{P}_{X}$ in $\mathbb{P}^{N^{*}}$, that is,

$$
X^{*}:=p_{2}\left(\mathscr{P}_{X}\right) \subseteq \mathbb{P}^{N^{*}} .
$$

The set $\mathscr{P}_{X}$ is easily seen to be a closed subset of $X \times \mathbb{P}^{N^{*}}$. For $x \in X_{\text {reg, }}$, we have $p_{1}^{-1}(x) \simeq\left(T_{x} X\right)^{*}=\mathbb{P}^{N-n-1} \subset \mathbb{P}^{N *}$. Then the set $\mathscr{P}_{X}$ is irreducible since

$$
p_{1}^{-1}\left(X_{\text {reg }}\right) \rightarrow X_{\text {reg }}
$$

is a $\mathbb{P}^{N-n-1}$-bundle. Therefore $\operatorname{dim}\left(\mathscr{P}_{X}\right)=N-1$, yielding $\operatorname{dim}\left(X^{*}\right) \leq N-1$.

Definition 2.2 (Dual defect). The dual defect of $X$, $\operatorname{def}(X)$, is defined as

$$
\operatorname{def}(X)=N-1-\operatorname{dim}\left(X^{*}\right) \geq 0 .
$$

To justify the name of conormal variety for $\mathscr{P}_{X}$ and to get some practice with the definitions, we refer to Exercise 9.8

A famous result of Landman, known as the Landman Parity Theorem, asserts that the dual defect of a smooth manifold $X^{n} \subset \mathbb{P}^{N}$ has the same parity as $\operatorname{dim}(X)=n$, if positive. 
3. Some examples revisited. We now describe some examples to become acquainted with these definitions.

EXAMPLE 3.1. It is easy to see that

$$
\text { Sing } v_{3}\left(\mathbb{P}^{1}\right)^{*}=\left\{[H] \in\left(\mathbb{P}^{3}\right)^{*} \mid H=T^{2} v_{3}\left(\mathbb{P}^{1}\right) \text { for some } p \in \nu_{3}\left(\mathbb{P}^{1}\right)\right\} \text {. }
$$

This also follows directly from the definition of Reflexivity, see Definition 3.3, and from Theorem 3.6 .

Let

$$
\begin{gathered}
v_{3}: \mathbb{P}^{1} \longrightarrow \mathbb{P}^{3}, \\
(t: s) \longmapsto\left(s^{3}: t s^{2}: t^{2} s: t^{3}\right) .
\end{gathered}
$$

Then

$$
T_{(t: s)}^{2} v_{3}\left(\mathbb{P}^{1}\right)=\mathbb{P}\left(\mathscr{L}\left(\frac{\partial^{2} v_{3}}{\partial t^{2}}, \frac{\partial^{2} v_{3}}{\partial t \partial s}, \frac{\partial^{2} v_{3}}{\partial s^{2}}\right)\right) .
$$

Let

$$
p=p(\widetilde{t})=\left(1: \widetilde{t}: \widetilde{t}^{2}: \widetilde{t}^{3}\right) .
$$

Then a direct computation shows that

$$
T_{p}^{2} v_{3}\left(\mathbb{P}^{1}\right): \widetilde{t}^{3} x_{0}-3 \widetilde{t}^{2} x_{1}+3 \widetilde{t} x_{2}-x_{3}=0
$$

and that

$$
\widetilde{C}=\left\{\left(-\widetilde{t}^{3}: 3 \widetilde{t}^{2}:-3 \widetilde{t}: 1\right)\right\}=\operatorname{Sing} v_{3}\left(\mathbb{P}^{1}\right)^{*} \stackrel{\text { proj. }}{\cong} v_{3}\left(\mathbb{P}^{1}\right) .
$$

Since

$$
T_{p(\widehat{t})} C^{\perp}=T_{p(\widetilde{t})} \widetilde{C}
$$

we deduce that

$$
\left(\nu_{3}\left(\mathbb{P}^{1}\right)^{*}=T \widetilde{C}=\bigcup_{q \in \widetilde{C}} T_{q} \widetilde{C}\right.
$$

so that $T \widetilde{C}$ is the degree- 4 surface $V(\Delta) \subset\left(\mathbb{P}^{3}\right)^{*}$.

ExAmPLE 3.2. Let $X=v_{4}\left(\mathbb{P}^{1}\right) \subset \mathbb{P}^{4}$ and let

$$
v_{4}\left(\mathbb{P}^{1}\right)=\overline{\left\{\left(1: t: t^{2}: t^{3}: t^{4}\right)\right\}} \subset \mathbb{P}^{4}
$$

where

$$
\begin{gathered}
v_{4}: \mathbb{P}^{1} \longrightarrow \mathbb{P}^{4}, \\
(s: t) \longmapsto\left(s^{4}: s^{3} t: s^{2} t^{2}: s t^{3}: t^{4}\right) .
\end{gathered}
$$

Then

$$
v_{4}\left(\mathbb{P}^{1}\right)^{*}=V\left(\Delta\left(a_{0}, \ldots, a_{4}\right)\right) \subset\left(\mathbb{P}^{4}\right)^{*}
$$

is a hypersurface of degree 6 (in general, $v_{d}\left(\mathbb{P}^{1}\right)^{*}$ has degree $2 d-2$ for all $d \geq 2$ ). Then

$$
\widetilde{C}=\left\{[H] \mid H=T_{p}^{3} C, p \in C\right\} \subset\left(\mathbb{P}^{4}\right)^{*}
$$

is projectively equivalent to $v_{4}\left(\mathbb{P}^{1}\right)$ by a calculation similar to that in Example 3.1 Notwithstanding, $\operatorname{Sing}\left(v_{4}\left(\mathbb{P}^{1}\right)^{*}\right)$ is bigger that $\widetilde{C}$. Indeed,

$$
\widetilde{C} \leftrightarrow H: H \cap C=4 p .
$$


Generically,

$$
H \cap C=\left\{p_{1}, p_{2}, p_{3}, p_{4}\right\},
$$

with $p_{i}$ 's distinct. We have also the following special cases:

1) $H \cap C=\left\{2 q, r_{1}, r_{2}\right\}$, with $H \supset T_{q} v_{4}\left(\mathbb{P}^{1}\right)$ but $H \not \supset T_{r_{i}} v_{4}\left(\mathbb{P}^{1}\right)$;

2) $H \cap C=\{2 q, 2 r\}$, with $H \supseteq\left\langle T_{q} C, T_{r} C\right\rangle=\mathbb{P}^{3}$, implying $H=\left\langle T_{q} C, T_{r} C\right\rangle$;

3) $H \cap C=\{3 q, r\}$ if and only if $H \supset T_{q}^{2} C, H \not \supset T_{r} C$.

Hyperplanes as in 2) and 3) produce singular points of $\nu_{4}\left(\mathbb{P}^{1}\right)^{*}$ not belonging to $\widetilde{C}$, see Example 4.8 for further details.

Definition 3.3 (Reflexive variety). A variety is said to be reflexive if the natural isomorphism between $\mathbb{P}^{N}$ and $\mathbb{P}^{N^{* *}}=\left(\mathbb{P}^{N^{*}}\right)^{*}$ induces an isomorphism between

$$
\mathscr{P}_{X} \subset X \times \mathbb{P}^{N^{*}} \subset \mathbb{P}^{N} \times \mathbb{P}^{N^{*}}
$$

and

$$
\mathscr{P}_{X^{*}} \subset X^{*} \times \mathbb{P}^{N^{* *}} \subset \mathbb{P}^{N^{*}} \times \mathbb{P}^{N^{* *}} \simeq \mathbb{P}^{N^{*}} \times \mathbb{P}^{N}
$$

For a reflexive variety, the natural identification between $\mathbb{P}^{N}$ and $\mathbb{P}^{N^{* *}}$ induces the equality $X=X^{* *}:=\left(X^{*}\right)^{*}$. See part (b) of Exercise 9.10 for a well-known example, considered firstly by Wallace, of a non-reflexive smooth plane curve $X \subset \mathbb{P}^{2}$ defined over a field of positive characteristic such that $X=X^{* *}$.

Let us take $[H] \in X^{*}$. By definition

$$
C_{H}:=C_{H}(X)=p_{2}^{-1}(H)=\overline{\left\{x \in X_{\mathrm{reg}} \mid T_{x} X \subseteq H\right\}}
$$

is precisely the closure of the non-singular points of $X$ where $H$ is tangent to $X$, the so-called contact locus of $H$ on $X$.

By definition $C_{H}$ is not empty so that $H \cap X$ is singular outside $\operatorname{Sing}(X)$ for $H \in X^{*}$. On the contrary if $[H] \notin X^{*}$, the hyperplane section $H \cap X$ can be singular only along $\operatorname{Sing}(X)$. This is the classical and well-known Bertini's Theorem, which we now state explicitly.

TheOrem 3.4 (Bertini's Theorem on hyperplane sections). Let $X \subset \mathbb{P}^{N}$ be a projective, irreducible non-degenerate variety of dimension $n=\operatorname{dim}(X)$. Then for every $H \in\left(\mathbb{P}^{N}\right)^{*} \backslash X^{*}$ the divisor $H \cap X$ is non-singular outside $\operatorname{Sing}(X)$.

In particular, if $X$ has at most a finite number of singular points $p_{1}, \ldots, p_{m}$, then for every $H \notin X^{*} \cup\left(p_{1}\right)^{*} \cup \ldots \cup\left(p_{m}\right)^{*}$, the hyperplane section $H \cap X$ is a non-singular subscheme of pure codimension one.

It can be shown that every hyperplane section of an irreducible variety of dimension $n \geq 2$ is connected. Thus for non-singular varieties with $n=\operatorname{dim}(X) \geq 2$, the hyperplane sections with hyperplanes $H \notin X^{*}$, being connected and non-singular, are also irreducible so that they are irreducible non-singular algebraic varieties.

For reflexive varieties $X \subset \mathbb{P}^{N}$ a general contact locus $C_{H}(X) \subset X$ is a linear space of dimension $\operatorname{def}(X)$. This is nothing but an interpretation of the isomorphism $X \simeq\left(X^{*}\right)^{*}$.

One should be careful in the interpretation of the result: it does not mean that the hyperplane remains tangent along the whole "contact locus", see Remark 8.7 and adapt it 
to the more general situation of a ruling of a cone. This is true only for non-singular varieties. In particular, reflexive varieties of positive dual defect contain positive dimensional families of linear spaces, imposing strong restrictions on their existence. We formalize this discussion by stating it for further reference in the text.

Proposition 3.5. Let $X \subset \mathbb{P}^{N}$ be a reflexive variety. Then for $[H] \in X_{\text {reg }}^{*}$,

$$
p_{1}\left(p_{2}^{-1}([H])\right)=\overline{\left\{x \in X_{\text {reg }} \mid T_{x} X \subset H\right\}}=\left(T_{[H]} X^{*}\right)^{\perp}=\mathbb{P}^{\operatorname{def}(X)} .
$$

The next result has an elementary and direct proof. It is considered a classical theorem, known at least to C. Segre. According to Kleiman it was also discovered in some form by Monge. The modern treatment pointing out the pathologies occurring in positive characteristic and stating it explicitly seems to be due to Wallace.

In Exercise 9.10 we show, following Wallace, several well-known examples of smooth irreducible non-degenerate varieties $X \subset \mathbb{P}^{N}$, whose associated map $p_{2}: \mathscr{P}_{X} \rightarrow X^{*}$ is not generically smooth so that $X$ cannot be reflexive. As we now prove, the condition of generic smoothness of $p_{2}$ is indeed equivalent to reflexivity.

THEOREM 3.6 (Reflexivity Theorem, Wallace). Let $X \subset \mathbb{P}^{N}$ be an irreducible nondegenerate projective variety. Then $X$ is reflexive if and only if $p_{2}: \mathscr{P}_{X} \rightarrow X^{*}$ is a generically smooth morphism.

In particular, if $\operatorname{char}(K)=0$, all projective irreducible varieties are reflexive.

Another natural and similar problem is to ask whether a general tangent space to a (smooth) variety $X$ is tangent to $X$ at more than one point. During the discussion we will always suppose $\operatorname{char}(K)=0$ to avoid the intriguing and strange pathologies in positive characteristic, which are usually variations on the themes described in Exercise 9.10

By Proposition 3.5 a general tangent space to an irreducible non-degenerate curve, not a line, is tangent only at one point. On the other hand if $X$ is a cone over a curve, we know that a general tangent space is tangent precisely along the ruling passing through the point. From this point of view the unique common feature of these two classes of irreducible varieties is the linearity of the locus of points at which a general tangent linear space remains tangent.

Definition 3.7 (Gauss maps). Let $X \subset \mathbb{P}^{N}$ be an irreducible projective variety of dimension $n=\operatorname{dim}(X) \geq 1$, let $m \geq n$ and let $\mathbb{G}(m, N)$ be the Grassmannian parametrizing linear subspaces of dimension $m$ in $\mathbb{P}^{N}$. Let

$$
\mathscr{P}_{X}^{m}:=\overline{\left\{\left((x,[L]) \in X_{\text {reg }} \times \mathbb{G}(m, N) \mid T_{x} X \subseteq L\right\}\right.} \subset X \times \mathbb{G}(m, N) .
$$

Let us consider the projections of $\mathscr{P}_{X}^{m}$ onto the factors $X$ and $\mathbb{G}(m, N)$,

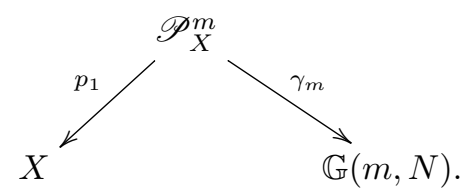

The variety of $m$-dimensional tangent subspaces to $X, X_{m}^{*}$, is the scheme-theoretic image of $\mathscr{P}_{X}^{m}$ in $\mathbb{G}(m, N)$, that is,

$$
X_{m}^{*}:=\gamma_{m}\left(\mathscr{P}_{X}^{m}\right) \subset \mathbb{G}(m, N)
$$


For $m=N-1$, we recover the dual variety and its definition, while for $m=n$, we get the usual Gauss map $\mathscr{G}_{X}: X \rightarrow \mathbb{G}(n, N)$ which associates to a point $x \in X_{\text {reg }}$ the point $\left[T_{x} X\right] \in \mathbb{G}(n, N)$, that is, $\mathscr{G}_{X}(x):=\gamma_{n}(x)=\left[T_{x} X\right]$ for $x \in X_{\text {reg. }}$.

If $X=V(f) \subset \mathbb{P}^{N}$ is a hypersurface, then $n=N-1$ and clearly the Gauss map

$$
\mathscr{G}_{X}: X \rightarrow \mathbb{P}^{N^{*}}=\mathbb{G}(N-1, N)
$$

associates to a smooth point $p$ of $X$ its tangent hyperplane. Thus in coordinates the Gauss map is given by the formula

$$
\mathscr{G}_{X}(p)=\left(\frac{\partial f}{\partial X_{0}}(p): \ldots: \frac{\partial f}{\partial X_{N}}(p)\right) .
$$

The following result is another interesting consequence of reflexivity, whose proof follows easily from Proposition 3.5 For smooth varieties several notable improvements are known applications of the so called Zak's Theorem on Tangency.

THEOREM 3.8 (Linearity of general contact loci for reflexive varieties). Let $X \subset \mathbb{P}^{N}$ be an irreducible projective non-degenerate reflexive variety of dimension $n=\operatorname{dim}(X) \geq 1$.

Then the general fiber of the morphism $\gamma_{m}: \mathscr{P}_{X}^{m} \rightarrow X_{m}^{*}$ is a linear space of dimension

$$
\operatorname{dim}\left(\mathscr{P}_{X}^{m}\right)-\operatorname{dim}\left(X_{m}^{*}\right) \text {. }
$$

In particular, the closure of a general fiber of $\mathscr{G}_{X}: X \rightarrow X_{n}^{*} \subset \mathbb{G}(n, N)$ is a linear space of dimension $n-\operatorname{dim}\left(\mathscr{G}_{X}(X)\right)$ so that a general linear tangent space is tangent along an open subset of a linear space of dimension $n-\operatorname{dim}\left(\mathscr{G}_{X}(X)\right)$.

\section{Dual curve of a projective curve after [8]. Let}

$$
C \subset \mathbb{P}^{N}
$$

be an irreducible projective algebraic curve over $\mathbb{C}$.

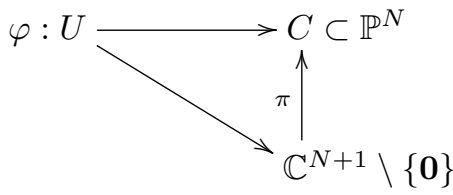

with $\varphi(U) \subseteq C_{\text {reg. }}$

$$
\operatorname{rk}(\varphi)=\max _{r \in \mathbb{N}}\left\{\varphi(s), \varphi^{\prime}(s), \ldots, \varphi^{r}(s) \in \mathbb{C}^{N+1} \text { are linearly independent on } \widetilde{U} \subseteq U\right\}
$$

We shall assume $\operatorname{rk}(\varphi)=N$, i.e., $C \subseteq \mathbb{P}^{N}$ is not degenerated, i.e., not contained in a hyperplane $H \subset \mathbb{P}^{N}$. For $0 \leq k \leq \operatorname{rk}(\varphi)=N$ we can define

$$
\begin{aligned}
& \varphi^{(k)}: U \longrightarrow \mathbb{G}(k, N)=G(k+1, N+1), \\
& s \longmapsto\left[\varphi(s) \wedge \ldots \wedge \varphi^{k}(s)\right] \in \mathbb{P} \Lambda^{k+1} \mathbb{C}^{N+1} .
\end{aligned}
$$

Definition 4.1. $\varphi^{k}(U) \subseteq \mathbb{G}(k, N)$ is called the $k$-associated curve of $C \subset \mathbb{P}^{N}$.

REMARK 4.2. $\mathbb{P}\left(\mathscr{L}\left(\varphi(s), \ldots, \varphi^{k}(s)\right)\right)=T_{\varphi(s)}^{k} C$ is the $k$-osculating space to $C$ at $\varphi(s)$.

If $k=N-1$, then

$$
U \stackrel{\varphi^{N-1}}{\longrightarrow} \mathbb{G}(N-1, N) \stackrel{D}{\cong}\left(\mathbb{P}^{N}\right)^{*}
$$




\section{DEFINITION 4.3.}

$$
\widetilde{\varphi}=D \circ \varphi^{N-1}: U \longrightarrow \widetilde{C} \subset\left(\mathbb{P}^{N}\right)^{*}
$$

is called the dual (or associated) curve to $C \subset \mathbb{P}^{N}$. A point $q \in \widetilde{C}$ represents an osculating hyperplane to $C \subset \mathbb{P}^{N}$.

ExAmple 4.4. Let $C=v_{3}\left(\mathbb{P}^{1}\right)=\overline{\left\{\left(1: t: t^{2}: t^{3}\right)\right\}} \subset \mathbb{P}^{3}$ and $\widehat{C}=\overline{\left\{\left(-t^{3}: 3 t^{2}:-3 t: 1\right)\right\}} \subset$ $\left(\mathbb{P}^{3}\right)^{*}$. We have a natural duality:

$$
D: \mathbb{G}(N-k-1, N) \longrightarrow \mathbb{G}^{*}(k, N)=\mathbb{G}\left(k,\left(\mathbb{P}^{N}\right)^{*}\right) .
$$

Let $\mathbb{P}^{N}=\mathbb{P}(V)$ and $\left(\mathbb{P}^{N}\right)^{*}=\mathbb{P}\left(V^{*}\right)$. If $L=\mathbb{P}(U)=\mathbb{P}^{N-k-1}$, then

$$
D(L)=\mathbb{P}(\operatorname{Ann}(U)),
$$

where

$$
\operatorname{Ann}(U)=\left\{\alpha \in V^{*}|\alpha|_{U} \equiv 0\right\}
$$

We have the diagram:

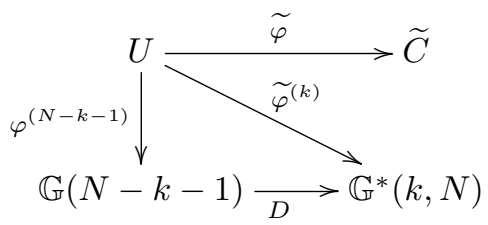

Under our hypotheses, for every $0 \leq k \leq N$ :

Proposition 4.5 ([8]). $\widetilde{\varphi}^{k}=D \circ \varphi^{(N-k-1)}$.

Proof. By induction $k=0: \widetilde{\varphi}=D \circ \varphi^{(N-1)}$.

$$
\varphi^{(k)}=\left(\widetilde{\varphi}^{(k-1)}\right)^{(1)}=\left(D \circ \varphi^{(N-(k-1)-1)}\right)^{(1)}=\left(D \circ \varphi^{(N-k)}\right)^{(1)}=D \circ \varphi^{(N-k-1)} .
$$

COROLlary 4.6. Identifying $\left(\mathbb{P}^{N}\right)^{*}$ with $\mathbb{P}^{N}$, we have

$$
\widetilde{\widetilde{C}}=C \text {. }
$$

If $k=N-2, \widetilde{\varphi}^{(N-1)}=D \circ \varphi^{(1)}$ and

$$
\varphi^{(1)}(s) \leftrightarrow T_{\varphi(s)} C, \quad \widetilde{\varphi}^{(N-2)}(s)=T_{\widetilde{\varphi}(s)}^{N-2} \widetilde{C}
$$

implies

$$
T_{\varphi(s)} C^{\perp}=T_{\widetilde{\varphi}(s)}^{N-2} \widetilde{C}
$$

and hence

$$
C^{*}=\overline{\bigcup_{s \in U} T_{\varphi(s)} C^{\perp}}=\overline{\bigcup_{s \in U} T_{\widetilde{\varphi}(s)}^{N-2} \widetilde{C}}=\operatorname{Tan}^{N-2} \widetilde{C} .
$$

For $N=3$,

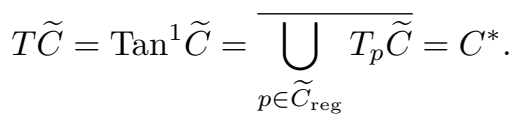

More generally,

$$
T_{\varphi(s)}^{k} C^{\perp}=T_{\widetilde{\varphi}(s)}^{N-k-1} \widetilde{C} .
$$


Important CONSEQUENCE. If $\widetilde{C} \subset\left(\mathbb{P}^{N}\right)^{*}$ is projectively equivalent to $C \subset \mathbb{P}^{N}$, the calculation of $C^{*}$ reduces essentially to that of $\operatorname{Tan}^{N-2} C$, a classically technique very well known to projective differential geometers.

EXAmPLE 4.7. Let $v_{3}\left(\mathbb{P}^{1}\right)^{*}=V(\Delta) \subset\left(\mathbb{P}^{3}\right)^{*}$. Recall that

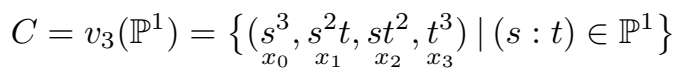

is the set of the solutions of the system

$$
\begin{cases}x_{0} x_{2}-x_{1}^{2}=0 & q_{0} \\ x_{0} x_{3}-x_{1} x_{2}=0 & q_{1} \\ x_{1} x_{3}-x_{2}^{2}=0 & q_{2}\end{cases}
$$

Let $p \in \mathbb{P}^{3} \backslash C$. By projecting $C$ onto a skew plane we obtain a plane rational irreducible cubic curve $C^{\prime} \subset \mathbb{P}^{2}$ which has a unique singular point $q$, which is a double point. This means that through $p$ there passes a unique secant line or a unique tangent line to $C$. In the first case $q$ will be a node, in the second case an ordinary cusp. From this perspective $T C$ is the closure of the locus of points of $\mathbb{P}^{3} \backslash C$ through which there passes a unique tangent line to $C$.

The quadratic equations $q_{0}, q_{1}, q_{2}$ are the $2 \times 2$ minors of the matrix

$$
\left[\begin{array}{lll}
x_{0} & x_{1} & x_{2} \\
x_{1} & x_{2} & x_{3}
\end{array}\right]
$$

We obviously have

$$
0=\left|\begin{array}{ccc}
x_{0} & x_{1} & x_{2} \\
x_{0} & x_{1} & x_{2} \\
x_{1} & x_{2} & x_{3}
\end{array}\right|=x_{0} q_{2}-x_{1} q_{1}+x_{2} q_{0}
$$

and

$$
0=\left|\begin{array}{lll}
x_{1} & x_{2} & x_{3} \\
x_{0} & x_{1} & x_{2} \\
x_{1} & x_{2} & x_{3}
\end{array}\right|=x_{1} q_{2}-x_{2} q_{1}+x_{3} q_{0}
$$

Consider the system

$$
\left\{\begin{array}{l}
x_{0} q_{2}-x_{1} q_{1}+x_{2} q_{0}=0 \\
x_{1} q_{2}-x_{2} q_{1}+x_{3} q_{0}=0
\end{array}\right.
$$

which can be rewritten in the form

$$
\left[\begin{array}{cccc}
q_{2} & -q_{1} & q_{0} & 0 \\
0 & q_{2} & -q_{1} & q_{0}
\end{array}\right] \cdot\left[\begin{array}{l}
x_{0} \\
x_{1} \\
x_{2} \\
x_{3}
\end{array}\right]=\left[\begin{array}{l}
0 \\
0
\end{array}\right]
$$

Let

$$
A=\left[\begin{array}{cccc}
q_{2} & -q_{1} & q_{0} & 0 \\
0 & q_{2} & -q_{1} & q_{0}
\end{array}\right]
$$

Then for every $p \in \mathbb{P}^{3}$ we have

$$
\operatorname{rk}(A(p))=1 \Longleftrightarrow p \in C .
$$


Suppose now that $p \in \mathbb{P}^{3} \backslash C$ and consider the system

$$
A(p) \cdot\left[\begin{array}{l}
x_{0} \\
x_{1} \\
x_{2} \\
x_{3}
\end{array}\right]=\left[\begin{array}{l}
0 \\
0
\end{array}\right],
$$

whose solutions represent a line $L_{p} \subset \mathbb{P}^{3}$. Moreover, clearly $p \in L_{p}$. Let us now calculate $L_{p} \cap C$. It is sufficient to substitute the parametrization $C=\nu_{3}\left(\mathbb{P}^{1}\right)$ into (4) obtaining the system

$$
\left\{\begin{array}{l}
s^{3} q_{2}(p)-s^{2} t q_{1}(p)+s t^{2} q_{0}(p)=0 \\
s^{2} t q_{2}(p)-s t^{2} q_{1}(p)+t^{3} q_{0}(p)=0 .
\end{array}\right.
$$

We can suppose $s \cdot t \neq 0$ and we get a single equation

$$
s^{2} q_{2}(p)-s t q_{1}(p)+t^{2} q_{0}(p)=0 .
$$

The solutions of (5) substituted into $\nu_{3}(s, t)$ give the points of intersections $L_{p} \cap \nu_{3}\left(\mathbb{P}^{1}\right)$. In particular, $L_{p} \cap \nu_{3}\left(\mathbb{P}^{1}\right) \neq \emptyset$ and the line $L_{p}$ is either a secant or a tangent line to $\nu_{3}\left(\mathbb{P}^{1}\right)$. The line $L_{p}$ is tangent to $\nu_{3}\left(\mathbb{P}^{1}\right)$ if and only if

$$
q_{1}^{2}(p)-4 q_{0}(p) q_{2}(p)=0 .
$$

In conclusion, we proved that

$$
\begin{aligned}
T C & =V\left(q_{0} q_{2}-4 q_{1}^{2}\right) \\
& =V\left(x_{0}^{2} x_{3}^{2}-6 x_{0} x_{1} x_{2} x_{3}-3 x_{1}^{2} x_{2}^{2}+4 x_{1}^{3} x_{3}+4 x_{0} x_{2}^{3}\right) .
\end{aligned}
$$

Let

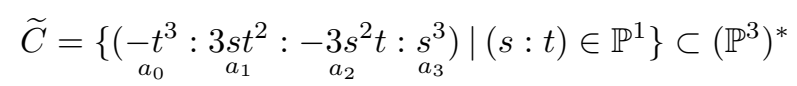

be the dual curve of $C$.

The equalities

$$
\left\{\begin{array}{l}
a_{0}=-x_{3} \\
a_{1}=3 x_{2} \\
a_{2}=-3 x_{1} \\
a_{3}=x_{0}
\end{array}\right.
$$

imply

$$
C^{*}=T \widetilde{C}=V\left(27 a_{0}^{2} a_{3}^{2}-18 a_{0} a_{1} a_{2} a_{3}-a_{1}^{2} a_{2}^{2}+4 a_{0} a_{2}^{3}+4 a_{3} a_{1}^{3}\right)=V(-\Delta) .
$$

We shall generalize this result to $\operatorname{LG}(3,6)$ later following strictly the same path.

EXAMPLE $4.8\left(v_{4}\left(\mathbb{P}^{1}\right)^{*}\right.$ revisited $)$. Let

$$
C=v_{4}\left(\mathbb{P}^{1}\right) \subset T C .
$$

Both $C$ and $T C$ are contained in

$$
S C=\overline{\bigcup_{p_{i} \in C, p_{1} \neq p_{2}}\left\langle p_{1}, p_{2}\right\rangle}
$$


and also in $\operatorname{Tan}^{2} C$. Then

$$
\widetilde{C} \subset T \widetilde{C} \subset \operatorname{Tan}^{2} \widetilde{C}=C^{*}
$$

and

$$
T \widetilde{C} \subset S \widetilde{C}
$$

Then a general point of $(S C)^{*}$ corresponds to a hyperplane $H \subset \mathbb{P}^{4}$ such that

$$
H \cap C=\left\{2 p_{1}, 2 p_{2}\right\},
$$

while $T \widetilde{C}$ to the $H^{\prime}$ 's such that $H \cap C=\{3 p, r\}$ because $T_{\widetilde{\varphi}(s)} \widetilde{C}=\left(T_{\varphi(s)}^{2} C\right)^{\perp}$.

EXAMPLE 4.9. Let

$$
\begin{aligned}
& p=\left(\stackrel{x_{0}}{1}: \underset{T_{p}}{\mathbf{0}}: \ldots: \mathbf{0}:{\stackrel{x_{N(n)}}{0}}^{x}\right) \in \operatorname{LG}(n, 2 n) \subset \mathbb{P}^{N(n)}, \\
& H: \sum_{i=0}^{N(n)} a_{i} x_{i}=0 .
\end{aligned}
$$

Then

$$
\begin{aligned}
p \in H & \Leftrightarrow a_{0}=0, \\
H \supset T_{p} H & \Leftrightarrow a_{0}=a_{1}=\ldots=a_{n(n+1) / 2}=0, \\
i \geq 2, H \supset T_{p}^{i} H & \Leftrightarrow \text { coefficients } a_{j} \text { of the minors of order } \leq i \text { are zero. }
\end{aligned}
$$

Finally,

$H \supset T_{p}^{n-2} X \Leftrightarrow H \cap X$ projects birationally onto a hyperplane in the space

$$
(0: 0: \ldots: B: \beta) \text {. }
$$

The projection of $X$ from $T_{p}^{n-2} X$ has expression

$$
\left(0: 0: \ldots: A^{\#}: \operatorname{det}(A)\right) \text {. }
$$

Then

$$
\left(0: 0: \ldots: \frac{A^{\#}}{\operatorname{det}(A)}: 1\right)=\left(0: 0: \ldots: A^{-1}: 1\right),
$$

so that equation (7) shows that the Monge-Ampère equation corresponding to $H$ is linearizable.

EXAmple 4.10. Let $\mathbb{G}(1, N)=G(2, N+1), \mathbb{P}^{N}=\mathbb{P}(V)$, and $\Lambda^{2} V$ the antisymmetric matrices $(N+1) \times(N+1)$. Then

$$
\mathbb{G}(1, N) \subset \mathbb{P}\left(\Lambda^{2} V\right), \quad \operatorname{dim} \Lambda^{2} V=\frac{1}{2} N(N+1)
$$

is the Plücker embedding. Let us remark that

$$
N+1 \text { even } \Leftrightarrow N \text { odd; }
$$

$\operatorname{rk}(A)$ is always even for $A \in \Lambda^{2} V$.

If $N+1$ is even,

$$
\operatorname{det}(A)=\operatorname{Pf}(A)^{2}, \quad \operatorname{deg} \operatorname{Pf}=\frac{N+1}{2}
$$


and

$$
V(\mathrm{Pf})=\mathbb{G}(1, N)^{*} \subset \mathbb{P}\left(\Lambda^{2} V\right)^{*}
$$

is a hypersurface of degree $\frac{N+1}{2}$ (matrices of rank $<N+1 \Rightarrow 2 k \leq N-1$ ).

In particular,

$$
\begin{gathered}
\mathbb{G}(1,3)^{*}=K^{*} \subset \mathbb{P}^{4} \text { is a quadric hypersurface (Klein); } \\
\mathbb{G}(1,5)^{*} \subset\left(\mathbb{P}^{14}\right)^{*} \text { is a cubic hypersurface, etc. etc.; } \\
\mathbb{G}(1,4)^{*} \subset \mathbb{P}^{9} \text { and } \mathbb{G}(1,4)^{*} \stackrel{\text { proj. }}{\cong} \mathbb{G}(1,4)
\end{gathered}
$$

since for a $5 \times 5$ matrix $A$ we have $\operatorname{rk}(A)<4 \Rightarrow \operatorname{rk}(A)=2$. Moreover, $\operatorname{def}(\mathbb{G}(1,4))=$ $8-6=2$.

In general,

$$
\operatorname{def}(\mathbb{G}(1, N))= \begin{cases}0 & N \text { odd } \\ 2 & N \text { even. }\end{cases}
$$

Indeed, let us consider $\operatorname{rk}(A)$. If $N$ is odd, then $G(1, N)^{*}=V(\mathrm{Pf})$ is a hypersurface; if $N$ is even, $N+1$ is odd and $\mathbb{G}(1, N)^{*}=\{[B] \mid \operatorname{rk}(B)<N\}$ has codimension 3 in $\mathbb{P}\left(\Lambda^{2} V\right)^{*}$, that is, $\mathbb{G}(1, N)^{*}$ is the biggest possible closed orbit in $\mathbb{P}\left(\Lambda^{2} V\right)^{*}$.

EXAmple 4.11 (Segre variety $X=\mathbb{P}^{1} \times \mathbb{P}^{n-1} \subset \mathbb{P}^{2 n-1}, n \geq 2$ ).

$$
\begin{array}{r}
\mathbb{P}^{2 n-1}=\mathbb{P}\left(\mathscr{M}_{2 \times n}(\mathbb{C})\right) \supset\{[A] \mid \operatorname{rk}(A)=1\} \\
=\left\{[A] \mid A=\left[\begin{array}{l}
\alpha_{0} \\
\alpha_{1}
\end{array}\right]\left[\begin{array}{lll}
\beta_{0} & \ldots & \beta_{n-1}
\end{array}\right]\right\} \\
A=\left[\begin{array}{lll}
x_{0} & \ldots & x_{n-1} \\
x_{n} & \ldots & x_{2 n-1}
\end{array}\right] \\
\begin{cases}x_{i}=\alpha_{0} \beta_{i}, & i=0, \ldots, n-1 \\
x_{n+j}=\alpha_{1} \beta_{j}, & j=0, \ldots, n-1 .\end{cases}
\end{array}
$$

Let

$$
\mathbb{P}^{1} \times \mathbb{P}^{n-1} \stackrel{\varphi}{\stackrel{\varphi}{\longrightarrow}} \mathbb{P}^{2 n-1}
$$

Then $\varphi$ is an embedding and we can identify $\mathbb{P}^{1} \times \mathbb{P}^{n-1}$ with

$$
\varphi\left(\mathbb{P}^{1} \times \mathbb{P}^{n-1}\right) \subset \mathbb{P}^{2 n-1} .
$$

Then $\mathbb{P}^{1} \times \mathbb{P}^{n-1}$ is an homogeneous space so that $\left(\mathbb{P}^{1} \times \mathbb{P}^{n-1}\right)^{*}$ is also a closed orbit of $\left(\mathbb{P}^{2 n-1}\right)^{*}$, yielding that $\left(\mathbb{P}^{1} \times \mathbb{P}^{n-1}\right)^{*}$ is projectively equivalent to $\mathbb{P}^{1} \times \mathbb{P}^{n-1}$. In particular

$$
\operatorname{dim}\left(\left(\mathbb{P}^{1} \times \mathbb{P}^{n-1}\right)^{*}\right)=n=\operatorname{dim}\left(\mathbb{P}^{1} \times \mathbb{P}^{n-1}\right) .
$$

5. $L G(3,6) \subset \mathbb{P}^{13}$ as twisted cubic over the algebra of $3 \times 3$ symmetric matrices. Let

$$
\begin{aligned}
& \mathrm{LG}(3,6)=\overline{\left\{\left(\underset{\alpha}{1}: \underset{A}{A}: A_{B}^{\sharp}: \operatorname{det}(A)\right) \mid A \in \operatorname{Sym}_{3 \times 3}(\mathbb{C})\right\}} \\
& \subset \mathbb{P}\left(\mathbb{C} \oplus \operatorname{Sym}_{3 \times 3}(\mathbb{C}) \oplus \operatorname{Sym}_{3 \times 3}(\mathbb{C}) \oplus \mathbb{C}\right)=\mathbb{P}^{13} .
\end{aligned}
$$


Suppose $\operatorname{det}(A) \neq 0$. Recall $A^{\sharp} \cdot A=\operatorname{det}(A) \mathrm{I}_{3 \times 3}=A \cdot A^{\sharp}$ and that

$$
\left(A^{\sharp}\right)^{\sharp}=\operatorname{det}(A) A \text {. }
$$

Then

$$
\left(1: A: A^{\sharp}: \operatorname{det}(A)\right)=\left(\frac{1}{\operatorname{det}(A)}: \frac{A}{\operatorname{det}(A)}: \frac{A^{\sharp}}{\operatorname{det}(A)}: 1\right) \text {. }
$$

Since $\frac{A^{\sharp}}{\operatorname{det}(A)}=A^{-1}$ and $\frac{A}{\operatorname{det}(A)} \cdot A^{\sharp}=I_{3 \times 3}$, we deduce that $\frac{A}{\operatorname{det}(A)}=\left(A^{\sharp}\right)^{-1}$.

EXERCISE 5.1. $\left(A^{\sharp}\right)^{-1}=\left(A^{-1}\right)^{\sharp}$.

Proposition 5.2.

$$
\begin{aligned}
\mathrm{LG}(3,6)= & \overline{\left\{\left(1: A: A^{\sharp}: \operatorname{det}(A)\right) \mid A \in \operatorname{Sym}_{3 \times 3}(\mathbb{C})\right\}} \\
= & \underbrace{\left\{\left(1: A: A^{\sharp}: \operatorname{det}(A)\right) \mid A \in \operatorname{Sym}_{3 \times 3}(\mathbb{C})\right\}}_{:=U} \\
& \cup \underbrace{\left\{\left(\operatorname{det}(B): B^{\sharp}: B: 1\right) \mid B \in \operatorname{Sym}_{3 \times 3}(\mathbb{C})\right\}}_{:=V},
\end{aligned}
$$

that is, on $U \cap V \cap\{\operatorname{det}(A) \neq 0\} \cap\{\operatorname{det}(B) \neq 0\}, B=A^{-1}$ is the change of coordinates between the two open sets $U$ and $V$.

Proof. Exercise.

$\mathrm{LG}(3,6)$ is a twisted cubic on $\operatorname{Sym}_{3 \times 3}(\mathbb{C})$ in the sense that its parametrization recalls that of a twisted cubic:

$$
v_{3}\left(\mathbb{P}^{1}\right)=\overline{\left\{\left(1: t: t^{2}: t^{3}\right)\right\}} .
$$

The manifold $\operatorname{LG}(3,6) \subset \mathbb{P}^{13}$ is defined, in the coordinates $(\alpha: A: B: \beta)$, by the following quadratic equations:

$$
\begin{aligned}
B^{\sharp} & =\alpha A, \\
A^{\sharp} & =\beta B, \\
A \cdot B & =\alpha \beta \mathrm{I}_{3 \times 3} .
\end{aligned}
$$

Let

$$
P(\alpha: A: B: \beta)=A^{\sharp} \cdot B^{\sharp}-\beta \operatorname{det}(A)-\alpha \operatorname{det}(B)-\frac{1}{4}(\operatorname{tr}(A \cdot B)-\alpha \beta)^{2},
$$

which is a homogeneous polynomial of degree 4 .

Proposition 5.3. With the previous notation we have

$$
V(P)=T \operatorname{LG}(3,6)=\bigcup_{p \in \operatorname{LG}(3,6)} T_{p} \mathrm{LG}(3,6) \subset \mathbb{P}^{13} .
$$

Proof. We only sketch the proof. For more details we refer to [5]:

a) $P$ is invariant for the action of a subgroup of $\mathrm{Sp}_{6}$.

b) $T_{(1: 0: 0: 0)} \mathrm{LG}(3,6)=\{(1: A: 0: 0)\}$ implies that $T_{(1: 0: 0: 0)} \mathrm{LG}(3,6) \subseteq V(P)$.

From part a) and b) the claim easily follows. 
Proposition 5.4. Let notation be as above and let $q=\left(1: A: A^{\sharp}: \operatorname{det}(A)\right) \in \mathrm{LG}(3,6)$. Then $T_{q}^{2} \mathrm{LG}(3,6) \subset \mathbb{P}^{13}$ is a hyperplane whose coordinates in $\mathbb{P}^{13^{*}}$ are

$$
\left(-\operatorname{det}(A), A^{\sharp}:-A: 1\right) \text {. }
$$

Proof. Explicit calculation using the parametrization $\left(\alpha^{3}: \alpha^{2} A: \alpha A^{\sharp}: \operatorname{det}(A)\right)$, Laplace and Euler formulas.

Let

$$
\widetilde{\mathrm{LG}(3,6)}=\overline{\left\{\left(-\operatorname{det}(A): A^{\sharp}:-A: 1\right)\right\}} \subset\left(\mathbb{P}^{13}\right)^{*}
$$

be the dual Lagrangian Grassmannian of $\mathrm{LG}(3,6) \subset \mathbb{P}^{13}$.

Since $T_{q}^{2} \mathrm{LG}(3,6)=q^{\perp}$ for the non-degenerate skew-symmetric form on $V=\mathbb{C}^{14}$ (where $\mathbb{P}(V)=\mathbb{P}^{13}$ ) defined by (8) and since $T_{q} \mathrm{LG}(3,6) \subset q^{\perp}$, we see that

(i) $\operatorname{LG}(3,6) \subset \mathbb{P}^{13}$ is a Legendrian subvariety;

(ii) $T_{q} \mathrm{LG}(3,6)^{\perp}=T_{\left(-\operatorname{det}(A): A^{\sharp}:-A: 1\right)} \widetilde{\mathrm{LG}(3,6)}$.

From (ii) we deduce the key formula

$$
\mathrm{LG}(3,6)^{*}=T \widetilde{\mathrm{LG}(3,6)}=\underbrace{\bigcup}_{p \in \mathrm{LG}(3,6)} T_{p} \widetilde{\mathrm{LG}(3,6)}
$$

and that $\operatorname{LG}(3,6)^{*}$ is thus a quartic hypersurface obtained from $V(P)$ by the linear change of coordinates imposed by (8). Thus to compute $\mathrm{LG}(3,6)^{*}$ we followed the same ideas used in Example 4.7 and leading to equation (6). Then, we can deduce, letting $(\widetilde{\alpha}: \widetilde{A}: \widetilde{B}: \widetilde{\beta})$ be the dual coordinates of $(\alpha: A: B: \beta)$,

$$
\mathrm{LG}(3,6)^{*}=V(\widetilde{P}) \subset \mathbb{P}^{13^{*}}
$$

with

$$
\begin{aligned}
\widetilde{P}(\widetilde{\alpha}: \widetilde{A}: \widetilde{B}: \widetilde{\beta})=P(\widetilde{\beta}, & -\widetilde{B}, \widetilde{A},-\widetilde{\alpha}) \\
& =(-\widetilde{B})^{\sharp} \cdot \widetilde{A^{\sharp}}-\widetilde{\alpha} \operatorname{det}(\widetilde{B})-\widetilde{\beta} \operatorname{det}(\widetilde{A})-\frac{1}{4}(\operatorname{tr}(\widetilde{A} \cdot \widetilde{B})-\widetilde{\alpha} \cdot \widetilde{\beta})^{2} .
\end{aligned}
$$

REMARK 5.5. By Theorem 1.5 a non-degenerate Monge-Ampère equation of dimension 3 is integrable if and only if

$$
[H] \in V(\widetilde{P})_{\mathrm{reg}}=T \widetilde{\mathrm{LG}(3,6)_{\mathrm{reg}}}=\widetilde{\mathrm{LG}(3,6)_{\mathrm{reg}}} \subset\left(\mathbb{P}^{13}\right)^{*} .
$$

\section{Tangent cones and tangent spaces of an algebraic variety and their asso-} ciated varieties. Let $X$ be an algebraic variety, or more generally a scheme of finite type, over a fixed algebraically closed field $K$. Let $x \in X$ be a closed point. We briefly recall the definitions of tangent cone to $X$ at $x$ and of tangent space to $X$ at $x$. For more details one can consult [11.

Definition 6.1 (Tangent cone at a point). Let $U \subset X$ be an open affine neighborhood of $x$, let $i: U \rightarrow \mathbb{A}^{N}$ be a closed immersion and let $U$ be defined by the ideal $I \subset K\left[X_{1}, \ldots, X_{N}\right]$. There is no loss of generality in supposing $i(x)=(0, \ldots, 0) \in \mathbb{A}^{N}$. Given $f \in K\left[X_{1}, \ldots, X_{N}\right]$ with $f(0, \ldots, 0)=0$, we can define the leading form (or 
initial form/term) $f^{\text {in }}$ of $f$ as the non-zero homogeneous polynomial of lowest degree in its expression as a sum of homogenous polynomials in the variables $X_{i}$ 's. Let

$I^{\text {in }}=\left\{\right.$ the ideal generated by the leading form (or initial term) $f^{\text {in }}$ of all $\left.f \in I\right\}$.

Then

$$
C_{x} X:=\operatorname{Spec}\left(\frac{K\left[X_{1}, \ldots, X_{N}\right]}{I^{\text {in }}}\right),
$$

is called the affine tangent cone to $X$ at $x$.

The previous definition does not depend on the choice of $U \subset X$ or on the choice of $i: U \rightarrow \mathbb{A}^{N}$. Indeed, letting $\left(\mathscr{O}_{x}, m_{x}\right)$ be the local ring of regular functions of $X$ at $x$, one immediately verifies that

$$
\frac{k\left[X_{1}, \ldots, X_{N}\right]}{I^{\text {in }}} \simeq \operatorname{gr}\left(\mathscr{O}_{x}\right):=\bigoplus_{n \geq 0} \frac{m_{x}^{n}}{m_{x}^{n+1}},
$$

yielding an intrinsic expression for the $K$-algebra defining $C_{x} X$.

The previous isomorphism assures that we can calculate $C_{x} X$ by choosing an arbitrary open neighborhood $U$ of $x$ and moreover that the definition is local on $X$. It should be observed that $C_{x} X$ is a scheme, which can be neither irreducible nor reduced as shown by easy examples, e.g. a cubic plane curve with a node, respectively a cubic plane curve with a cusp. We now obtain a geometrical interpretation of this cone and find some of its properties.

Since $C_{x} X$ is locally defined by homogeneous forms, it can be naturally projectivized and thought of as a subscheme of $\mathbb{P}^{N-1}=\mathbb{P}\left(\mathbb{A}^{N}\right)$. If we consider the blow-up of $x \in U \subset \mathbb{A}^{N}, \pi: \mathrm{Bl}_{x} U \rightarrow U$, then $\mathrm{Bl}_{x} U$ is naturally a subscheme of $U \times \mathbb{P}^{N-1} \subset$ $\mathbb{A}^{N} \times \mathbb{P}^{N-1}$ and the exceptional divisor $E:=\pi^{-1}(x)$ is naturally a subscheme of $x \times \mathbb{P}^{N-1}$. With these identifications one shows that $E \simeq \mathbb{P}\left(C_{x} X\right) \subset \mathbb{P}^{N-1}$ as schemes. In particular, if $X$ is equidimensional at $x$, then $C_{x} X$ is an equidimensional scheme of dimension $\operatorname{dim}(X)$ because $E$ is a Cartier divisor on $\mathrm{Bl}_{x} U$.

If $X \subset \mathbb{P}^{N}$ is quasi-projective, we define the projective tangent cone to $X$ at $x$, indicated by $\mathbf{C}_{x} X$, as the closure of $C_{x} X \subset \mathbb{A}^{N}$ in $\mathbb{P}^{N}$, where $x \in U=\mathbb{A}^{N} \cap X$ is a suitable chosen affine neighborhood.

We now recall the definition of tangent space to $X$ at $x \in X$.

DEFINITION 6.2 (Tangent space at a point, tangent variety to a variety). Let the notation be as in the previous definition and let $\mathbf{0}=(0, \ldots, 0) \in \mathbb{A}^{N}$. Given $f \in K\left[X_{1}, \ldots, X_{N}\right]$ with $f(\mathbf{0})=0$, we can define the linear term $f^{\text {lin }}$ of $f$ as the degree one homogeneous polynomial in its expression as a sum of homogenous polynomials in the variables $X_{i}$ 's. If the degree one term is zero we define $f^{\text {lin }}=0$. In other words,

$$
f^{\operatorname{lin}}=\sum_{i=1}^{N} \frac{\partial f}{\partial X_{i}}(\mathbf{0}) X_{i} .
$$

Let

$$
I^{\text {lin }}=\left\{\text { the ideal generated by the linear terms } f^{\text {lin }} \text { of all } f \in I\right\} .
$$


Then

$$
t_{x} X:=\operatorname{Spec}\left(\frac{K\left[X_{1}, \ldots, X_{N}\right]}{I^{\operatorname{lin}}}\right)
$$

is called the affine tangent space to $X$ at $x$.

Geometrically it is the locus of tangent lines to $X$ at $x$, where a line through $x$ is tangent to $X$ at $x$ if it is tangent to the hypersurfaces $V(f)=0, f \in I$, i.e. if the multiplicity of intersection of the line with $V(f)$ at $(0, \ldots, 0)$ is greater than one for all $f \in I$. In particular, this locus is a linear subspace of $\mathbb{A}^{N}$, being an intersection of linear subspaces.

Since $I^{\text {lin }} \subseteq I^{\text {in }}$, we deduce the inclusion of schemes

$$
C_{x} X \subseteq t_{x} X
$$

and that $t_{x} X$ is a linear subspace of $\mathbb{A}^{N}$ containing $C_{x} X$ as a subscheme (and not only as a set). In particular, for every $x \in X \operatorname{dim}\left(t_{x} X\right) \geq \operatorname{dim}(X)$ holds.

We recall that a point $x \in X$ is non-singular if and only if $C_{x} X=t_{x} X$. Since $t_{x} X$ is reduced and irreducible and since $C_{x} X$ is of $\operatorname{dimension} \operatorname{dim}(X)$, we have that $x \in X$ is non-singular if and only if $\operatorname{dim}\left(t_{x} X\right)=\operatorname{dim}(X)$.

Once again there is an intrinsic definition of $t_{x} X$ since

$$
\frac{K\left[X_{1}, \ldots, X_{N}\right]}{I^{\operatorname{lin}}} \simeq S\left(\frac{m_{x}}{m_{x}^{2}}\right),
$$

where $S\left(m_{x} / m_{x}^{2}\right)$ is the symmetric algebra of the $K$-vector space $m_{x} / m_{x}^{2}$.

The natural surjection

$$
S\left(\frac{m_{x}}{m_{x}^{2}}\right) \rightarrow \operatorname{gr}\left(\mathscr{O}_{x}\right)=\bigoplus_{n \geq 0} \frac{m_{x}^{n}}{m_{x}^{n+1}}
$$

shows that $t_{x} X$ is the linear span of $C_{x} X$ inside $\mathbb{A}^{N}$, that is, the smallest linear space of $\mathbb{A}^{N}$ containing $C_{x} X$ as a subscheme (and not only as a set).

If $X \subset \mathbb{P}^{N}$ is a quasi-projective variety, we define the projective tangent space to $X$ at $x$, indicated by $T_{x} X$, as the closure of $t_{x} X \subset \mathbb{A}^{N}$ in $\mathbb{P}^{N}$, where $x \in U=\mathbb{A}^{N} \cap X$ is a suitable chosen affine neighborhood. Then $T_{x} X$ is a linear projective space which varies with $x \in X$ and clearly $\mathbf{C}_{x} X \subseteq T_{x} X$ as schemes. We also set, for a (quasi)-projective variety $X \subset \mathbb{P}^{N}$,

$$
T X=\bigcup_{x \in X} T_{x} X
$$

the variety of tangents, or the tangent variety of $X$.

At a non-singular point $x \in X \subset \mathbb{P}^{N}$, the equality $\mathbf{C}_{x} X=T_{x} X$ says that every tangent line to $X$ at $x$ is the limit of a secant line $\langle x, y\rangle$ with $y \in X$ approaching $x$.

An interesting question is to investigate what are the limits of the secant lines $\left\langle x_{1}, x_{2}\right\rangle$, $x_{i} \in X, x_{1} \neq x_{2}$, when the $x_{i}$ 's, $i=1,2$, approach a fixed $x \in X$. For a non-singular point $x \in X$, every tangent line to $X$ at $x$ arises in this way, but for singular points this is not the case. These limits generate a cone, the tangent star cone to $X$ at $x$, which contains but does not usually coincide with $\mathbf{C}_{x} X$. From now on we restrict ourselves to the projective setting and we will not treat local questions related to tangent star cones. Firstly we introduce the notion of secant variety to a variety $X \subset \mathbb{P}^{N}$. 
Definition 6.3 (Secant varieties to a variety). For simplicity let us suppose that $X \subset \mathbb{P}^{N}$ is a closed irreducible subvariety.

Let

$$
S_{X}^{0}:=\left\{\left(\left(x_{1}, x_{2}\right), z\right) \mid z \in\left\langle x_{1}, x_{2}\right\rangle\right\} \subset\left((X \times X) \backslash \Delta_{X}\right) \times \mathbb{P}^{N} .
$$

The set is closed in $\left.(X \times X) \backslash \Delta_{X}\right) \times \mathbb{P}^{N}$ so that, taken with the reduced scheme structure, it is a quasi-projective variety. We remark that, by definition, it is a $\mathbb{P}^{1}$-bundle over $(X \times X) \backslash \Delta_{X}$, which is irreducible, so that $S_{X}^{0}$ is an irreducible quasi-projective variety of $\operatorname{dimension} \operatorname{dim}\left(S_{X}^{0}\right)=2 \operatorname{dim}(X)+1$.

Let $S_{X}$ be its closure in $X \times X \times \mathbb{P}^{N}$. Then $S_{X}$ is an irreducible projective variety of dimension $2 \operatorname{dim}(X)+1$, called the abstract secant variety to $X$. Let us consider the projections of $S_{X}$ onto the factors $X \times X$ and $\mathbb{P}^{N}$,

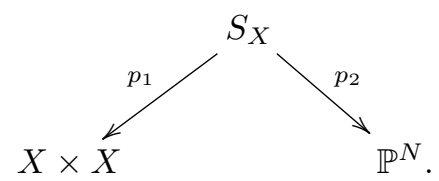

The secant variety to $X, S X$, is the scheme-theoretic image of $S_{X}$ in $\mathbb{P}^{N}$, i.e.

$$
S X=p_{2}\left(S_{X}\right)=\overline{\bigcup_{x_{1} \neq x_{2}, x_{i} \in X}\left\langle x_{1}, x_{2}\right\rangle} \subseteq \mathbb{P}^{N} .
$$

Thus $S X \subseteq \mathbb{P}^{N}$ is an irreducible projective variety of dimension

$$
s(X)=\operatorname{dim}(S X) \leq \min \{2 \operatorname{dim}(X)+1, N\},
$$

which geometrically is the variety swept out by the secant lines to $X$.

Let now $k \geq 1$ be a fixed integer. We can generalize the construction to the case of $(k+1)$-secant $\mathbb{P}^{k}$, i.e. to the variety swept out by the linear spaces generated by $k+1$ independent points on $X$.

Define

$$
\left(S_{X}^{k}\right)^{0} \subset \underbrace{X \times \ldots \times X}_{k+1} \times \mathbb{P}^{N}
$$

as the locally closed irreducible set

$$
\left(S_{X}^{k}\right)^{0}:=\left\{\left(\left(x_{0}, \ldots, x_{k}\right), z\right) \mid \operatorname{dim}\left(\left\langle x_{0}, \ldots, x_{k}\right\rangle\right)=k, z \in\left\langle x_{0}, \ldots, x_{k}\right\rangle\right\} .
$$

Let $S_{X}^{k}$, the abstract $k$-secant variety of $X$, be

$$
S^{k} X:=\overline{\left(S_{X}^{k}\right)^{0}} \subset \underbrace{X \times \ldots \times X}_{k+1} \times \mathbb{P}^{N} .
$$

The closed set $S_{X}^{k}$ is irreducible and of dimension $(k+1) \operatorname{dim}(X)+k$. Consider the projections of $S_{X}^{k}$ onto the factors $\underbrace{X \times \ldots \times X}_{k+1}$ and $\mathbb{P}^{N}$,

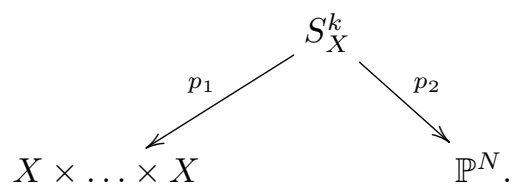


The $k$-secant variety to $X, S^{k} X$, is the scheme-theoretic image of $S_{X}^{k}$ in $\mathbb{P}^{N}$, i.e.

$$
S^{k} X=p_{2}\left(S_{X}^{k}\right)=\overline{\bigcup_{x_{i} \in X, \operatorname{dim}\left(\left\langle x_{0}, \ldots, x_{k}\right\rangle\right)=k}\left\langle x_{0}, \ldots, x_{k}\right\rangle} \subseteq \mathbb{P}^{N} .
$$

It is an irreducible algebraic variety of dimension

$$
s_{k}(X)=\operatorname{dim}\left(S^{k} X\right) \leq \min \{N,(k+1) \operatorname{dim}(X)+k\} .
$$

REMARK 6.4. Secant varieties have recently become very popular in connection with tensor decompositions and other problems coming from numerical analysis due to the work of Landsberg, Ottaviani, Chiantini and other authors. The notation commonly used by these specialists slightly differs from the one used here and usually $k$ is replaced by $k+1$. This recalls that we are dealing with $k+1$ general points on $X$ and they frequently use the symbol $\sigma^{k+1}(X)$ to indicate $S^{k} X$. The index $k$ in $S^{k} X$ reminds us that it is the locus of the $\mathbb{P}^{k}$ 's generated by $k+1$ general points on $X$.

Some authors define $k$-defective secant varieties as those for which $s_{k}(X)<\min \{N$, $(k+1) \operatorname{dim}(X)+k\}$ while others as those for which $s_{k}(X)<(k+1) \operatorname{dim}(X)+k$. We shall mainly be interested in the case $k=1$ here and we shall define secant defective varieties as those for which $s_{1}(X)=\operatorname{dim}(S X)<2 \operatorname{dim}(X)+1$, following the approach of Zak but not that of many other references cited in the bibliography. So, to avoid confusion, one should check the definition used in each reference consulted.

7. Join of varieties. We generalize to arbitrary irreducible varieties $X, Y \subset \mathbb{P}^{N}$ the notion of cone and the definition of the join of linear spaces.

Let us recall that if $L_{i} \simeq \mathbb{P}^{N_{i}} \subseteq \mathbb{P}^{N}, i=1,2$, is a linear subspace, then

$$
\left\langle L_{1}, L_{2}\right\rangle:=\bigcup_{x_{i} \in L_{i}, x_{1} \neq x_{2}}\left\langle x_{1}, x_{2}\right\rangle,
$$

is a linear space called the join of $L_{1}$ and $L_{2}$. It is the smallest linear subspace of $\mathbb{P}^{N}$ containing $L_{1}$ and $L_{2}$ so that $\left\langle L_{1}, L_{2}\right\rangle=\left\langle L_{1} \cup L_{2}\right\rangle$. From Grassmann's Formula we have

$$
\operatorname{dim}\left(\left\langle L_{1}, L_{2}\right\rangle\right)=\operatorname{dim}\left(L_{1}\right)+\operatorname{dim}\left(L_{2}\right)-\operatorname{dim}\left(L_{1} \cap L_{2}\right),
$$

where $\operatorname{dim}(\emptyset)=-1$ by definition. Grassmann's Formula shows that the dimension of the join depends on the intersection of the two linear spaces.

On the other hand, if $X \subset \mathbb{P}^{N} \subset \mathbb{P}^{N+1}$ is an irreducible subvariety and if $p \in \mathbb{P}^{N+1} \backslash \mathbb{P}^{N}$ is an arbitrary point, if we define as before

$$
S(p, X)=\bigcup_{x \in X}\langle p, x\rangle
$$

the cone of vertex $p$ over $X$, then for every $z \in\langle p, x\rangle, z \neq p$, we have by construction

$$
T_{z} S(p, X)=\left\langle p, T_{x} X\right\rangle=\left\langle T_{p} p, T_{x} X\right\rangle,
$$

showing the well-known fact that the tangent space to a cone is constant along its rulings.

As we shall see in the next section, once we have defined the join of two varieties we can linearize the problem looking at the tangent spaces and calculating the dimension of the join by studying the affine cones over the varieties, exactly as in the proof of the formula (9). The dimension of the join of two varieties will still depend on the 
intersection of the corresponding tangent spaces, a result known as Terracini's Lemma, [12. Moreover, a kind of property similar to the second tautological inequality in 10 will hold generically, at least in characteristic zero, see Theorem 8.1 .

DEFINITION 7.1 (Join of varieties; relative secant, tangent star and tangent varieties). Let $X, Y \subset \mathbb{P}^{N}$ be closed irreducible subvarieties.

Let

$$
S_{X, Y}^{0}:=\{(x, y, z), x \neq y \mid z \in\langle x, y\rangle\} \subset X \times Y \times \mathbb{P}^{N} .
$$

The set is locally closed so that, taken with the reduced scheme structure, it is a quasi-projective irreducible variety of dimension $\operatorname{dim}\left(S_{X, Y}^{0}\right)=\operatorname{dim}(X)+\operatorname{dim}(Y)+1$. Let $S_{X, Y}$ be its closure in $X \times Y \times \mathbb{P}^{N}$. Then $S_{X, Y}$ is an irreducible projective variety of $\operatorname{dimension} \operatorname{dim}(X)+\operatorname{dim}(Y)+1$, called the abstract join of $X$ and $Y$. Let us consider the projections of $S_{X, Y}$ onto the factors $X \times Y$ and $\mathbb{P}^{N}$,

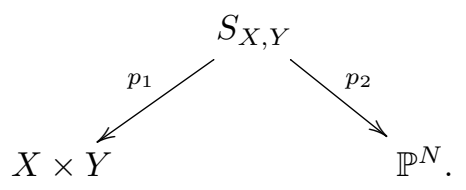

The join of $X$ and $Y, S(X, Y)$, is the scheme-theoretic image of $S_{X, Y}$ in $\mathbb{P}^{N}$, i.e.

$$
S(X, Y)=p_{2}\left(S_{X, Y}\right)=\overline{\bigcup_{x \neq y, x \in X, y \in Y}\langle x, y\rangle} \subseteq \mathbb{P}^{N} .
$$

Thus $S(X, Y) \subseteq \mathbb{P}^{N}$ is an irreducible projective variety of dimension

$$
s(X, Y)=\operatorname{dim}(S(X, Y)) \leq \operatorname{dim}(X)+\operatorname{dim}(Y)+1,
$$

swept out by lines joining points of $X$ with points of $Y$.

With this notation, after setting $X=S^{0}(X)$, we have $S(X, X)=S X$ and

$$
S\left(X, S^{k-1} X\right)=S^{k} X=S\left(S^{l} X, S^{h} X\right),
$$

if $h \geq 0, l \geq 0, h+l=k-1$. Moreover, for arbitrary irreducible varieties $X, Y$ and $Z$, we have

$$
S(X, S(Y, Z))=S(S(X, Y), Z),
$$

that is, join is an associative operation on the set of irreducible varieties of a fixed projective space.

When $Y \subseteq X \subset \mathbb{P}^{N}$ is an irreducible closed subvariety, the variety $S(Y, X)$ is usually called the relative secant variety of $X$ with respect to $Y$. Analogously,

$$
T(Y, X)=\bigcup_{y \in Y} T_{y} X
$$

We provide some immediate applications of the definition of join to properties of $S^{k} X$ and to characterizations of linear spaces. Let us recall that a variety $X \subset \mathbb{P}^{N}$ is said to be non-degenerate if $\langle X\rangle=\mathbb{P}^{N}$. 
Proposition 7.2 (Palatini). Let $X, Y \subset \mathbb{P}^{N}$ be closed irreducible subvarieties. Then:

1) for every $x \in X$,

$$
Y \subseteq S(x, Y) \subseteq T_{x} S(x, Y) \subseteq T_{x} S(X, Y)
$$

and in particular

$$
\langle x,\langle Y\rangle\rangle \subseteq T_{x} S(x, Y) .
$$

2) If $S^{k} X=S^{k+1} X$ for some $k \geq 0$, then $S^{k} X=\mathbb{P}^{s_{k}(X)} \subseteq \mathbb{P}^{N}$.

3) If $\operatorname{dim}\left(S^{k+1} X\right)=\operatorname{dim}\left(S^{k} X\right)+1$ for some $k \geq 0$, then $S^{k+1} X=\mathbb{P}^{s_{k+1}(X)}$ and $S^{k} X$ is a hypersurface in $\mathbb{P}^{s_{k+1}(X)}$.

4) Let $k$ be a nonnegative integer. If $S^{k+1} X \subset \mathbb{P}^{N}$ is not a linear space, then $S^{k} X \subseteq$ $\operatorname{Sing}\left(S^{k+1} X\right)$.

Thus to a non-degenerate irreducible closed subvariety $X \subset \mathbb{P}^{N}$ we can associate an ascending filtration of irreducible projective varieties, whose inclusions are strict by Proposition 7.2

$$
X=S^{0} X \subsetneq S X \subsetneq S^{2} X \subsetneq \ldots \subsetneq S^{k_{0}} X=\mathbb{P}^{N} .
$$

Therefore $k_{0}=k_{0}(X) \geq 1$ is well defined as the least integer $k \geq 1$ such that $S^{k} X=\mathbb{P}^{N}$.

The above immediate consequences of the definitions also give the next result, which was classically very well known.

Corollary 7.3 (Palatini). Let $C \subset \mathbb{P}^{N}$ be an irreducible non-degenerate projective curve. Then $s_{k}(C)=\min \{2 k+1, N\}$.

Let $X \subset \mathbb{P}^{N}$ be an irreducible non-degenerate projective variety of dimension $n \geq 1$ and let $k<k_{0}$. Then:

1) $s_{k}(X) \geq n+2 k$ for every $k<k_{0}$.

2) If $s_{j}(X)=n+2 j$ for some $j \geq 1, j<k_{0}$, then $s_{k}(X)=n+2 k$ for every $k \leq j$. In particular, if $s_{k}(X)=n+2 k$ for some $k \geq 1, k<k_{0}$, then $s(X)=n+2($ and $\left.S X \subsetneq \mathbb{P}^{N}\right)$.

Proof. For $k=0$ the assertion is true since $s_{0}(C)=\operatorname{dim}(C)=1$ by definition and we can argue by induction. Suppose $S^{k} C \varsubsetneqq \mathbb{P}^{N}$. By Proposition $7.2 s_{k}(C) \geq s_{k-1}(C)+2$ and the description $S^{k}(C)=S\left(C, S^{k-1} C\right)$ yields $s_{k}(C) \leq s_{k-1}(C)+2$. Combining the two inequalities we get $s_{k}(C)=s_{k-1}(C)+2=2(k-1)+1+2=2 k+1$, as claimed.

To prove the second part we argue as above. Thus, for $k<k_{0}, s_{k}(X) \geq s_{k-1}(X)+2=$ $n+2(k-1)+2=n+2 k$. Moreover, $s_{j}(X)=n+2 j$ yields $s_{k}(X)=n+2 k$ for every $k \leq j$, proving all the remaining claims.

8. Terracini's Lemma and its first applications. By definition the secant variety $S X$ of $X \subset \mathbb{P}^{N}$ is the join of $X$ with itself and it is not clear a priori how to calculate its dimension, see for example Exercise 9.3. More generally one would like to compute the dimension of $S(X, Y)$ for two arbitrary varieties $X, Y \subset \mathbb{P}^{N}$.

In fact, the circle of ideas, which allowed Terracini to solve the problem of calculating the dimension of $S X$, or more generally of $S^{k} X$, originated precisely from the study of examples like the ones considered in Exercise 9.3 and from the pioneering work of Scorza on secant defective varieties. 
To compute the dimension of $S(X, Y)$ in a simple way and to determine the relation between $T_{z} S(X, Y), T_{x} X$ and $T_{y} Y$, where $z \in\langle x, y\rangle, z \neq x, z \neq y, x \neq y$, we recall the definition of an affine cone over a projective variety $X \subset \mathbb{P}^{N}$.

Let $\pi: \mathbb{A}^{N+1} \backslash \mathbf{0} \rightarrow \mathbb{P}^{N}$ be the canonical projection. If $X \subset \mathbb{P}^{N}$ is a closed subvariety, we indicate by $C_{\mathbf{0}}(X)$ the affine cone over $X$, i.e.

$$
C_{\mathbf{0}}(X)=\pi^{-1}(X) \cup \mathbf{0} \subset \mathbb{A}^{N+1}
$$

is the affine variety cut out by the homogeneous polynomials in $N+1$ variables defining $X$ in $\mathbb{P}^{N}$. If $\mathbf{x} \neq \mathbf{0}$ is a point such that $\pi(\mathbf{x})=x \in X$, then

$$
\pi\left(t_{\mathbf{x}} C_{\mathbf{0}}(X)\right)=T_{x} X
$$

Moreover, if $L_{i}=\pi\left(U_{i}\right), i=1,2, U_{i}$ a vector subspace of $\mathbb{A}^{N+1}$, then by definition

$$
\left\langle L_{1}, L_{2}\right\rangle=\left\langle L_{1} \cup L_{2}\right\rangle=\pi\left(U_{1}+U_{2}\right),
$$

where $+: \mathbb{A}^{N+1} \times \mathbb{A}^{N+1} \rightarrow \mathbb{A}^{N+1}$ is the vector space operation. Therefore, regarded as a morphism of algebraic varieties, the differential of the sum coincides with the operation, that is,

$$
\begin{gathered}
d_{(\mathbf{x}, \mathbf{y})}: t_{(\mathbf{x}, \mathbf{y})}\left(\mathbb{A}^{N+1} \times \mathbb{A}^{N+1}\right)=t_{\mathbf{x}} \mathbb{A}^{N+1} \times t_{\mathbf{y}} \mathbb{A}^{N+1} \rightarrow t_{\mathbf{x}+\mathbf{y}} \mathbb{A}^{N+1} \\
(\mathbf{u}, \mathbf{v}) \mapsto \mathbf{u}+\mathbf{v} .
\end{gathered}
$$

With the above notation and definitions we deduce

$$
\overline{C_{\mathbf{0}}(X)+C_{\mathbf{0}}(Y)}=C_{\mathbf{0}}(S(X, Y)) .
$$

We are now in position to prove the so-called Terracini Lemma. The original proof of Terracini relies on the study of the differential of the second projection morphism $p_{2}: S_{X, Y} \rightarrow S(X, Y)$. Here we follow Ådlandsvik, [1], to avoid the difficulty, if any, of writing the tangent space at a point $(x, y, z) \in S_{X, Y}^{0}$. When writing $z \in\langle x, y\rangle$, we always suppose $x \neq y$.

TheOrem 8.1 (Terracini's Lemma). Let $X, Y \subset \mathbb{P}^{N}$ be irreducible subvarieties. Then:

1) for every $x \in X$, for every $y \in Y, x \neq y$, and for every $z \in\langle x, y\rangle$,

$$
\left\langle T_{x} X, T_{y} Y\right\rangle \subseteq T_{z} S(X, Y)
$$

2) if $\operatorname{char}(K)=0$, there exists an open subset $U$ of $S(X, Y)$ such that

$$
\left\langle T_{x} X, T_{y} Y\right\rangle=T_{z} S(X, Y)
$$

for every $z \in U, x \in X, y \in Y, z \in\langle x, y\rangle$. In particular

$$
\operatorname{dim}(S(X, Y))=\operatorname{dim}(X)+\operatorname{dim}(Y)-\operatorname{dim}\left(T_{x} X \cap T_{y} Y\right)
$$

for $x \in X$ and $y \in Y$ general points.

Proof. The first part follows from equation (8) and from the interpretation of the differential of the affine sum. The second part from generic smoothness applied to the affine cones over $X, Y$ and $S(X, Y)$.

Since we have quoted the original form given by Terracini, let us state it as an obvious corollary. 
Corollary $8.2([12])$. Let $X \subset \mathbb{P}^{N}$ be an irreducible subvariety of $\mathbb{P}^{N}$. Then:

1) for every $x_{0}, \ldots, x_{k} \in X$ and for every $z \in\left\langle x_{0}, \ldots, x_{k}\right\rangle$,

$$
\left\langle T_{x_{0}} X, \ldots, T_{x_{k}} X\right\rangle \subseteq T_{z} S^{k} X
$$

2) if $\operatorname{char}(K)=0$, there exists an open subset $U$ of $S^{k} X$ such that

$$
\left\langle T_{x_{0}} X, \ldots, T_{x_{k}} X\right\rangle=T_{z} S^{k} X
$$

for every $z \in U, x_{i} \in X, i=0, \ldots, k, z \in\left\langle x_{0}, \ldots, x_{k}\right\rangle$. In particular,

$$
\operatorname{dim}(S X)=2 \operatorname{dim}(X)-\operatorname{dim}\left(T_{x} X \cap T_{y} X\right)
$$

for $x, y \in X$ general points.

As an application we reinterpret Terracini's Lemma as the tangency of the tangent space to higher secant varieties at a general point along the locus described on $X$ by the secant spaces passing through the point.

Definition 8.3 (Tangency along a subvariety). Let $Y \subset X$ be a closed (irreducible) subvariety of $X$ and let $L=\mathbb{P}^{l} \subset \mathbb{P}^{N}, l \geq \operatorname{dim}(X)$, be a linear subspace.

The linear space $L$ is said to be tangent to $X$ along $Y$ if for every $y \in Y$

$$
T_{y} X \subseteq L
$$

i.e. if and only if $T(Y, X) \subseteq L$.

Clearly if $L$ is tangent to $X$ along $Y$, it is also $J$-tangent to $X$ along $Y$ and if $L$ is $J$-tangent to $X$ along $Y$ it is also $J$-tangent to $X$ with respect to $Y$.

In the case $L=\mathbb{P}^{N-1}$, the scheme-theoretic intersection $L \cap X=D$ is a divisor, i.e. a subscheme of pure dimension $\operatorname{dim}(X)-1$. By definition, for every $y \in D$, we have $T_{y} D=T_{y} X \cap L$ so that, if $X$ is a smooth variety, $L=\mathbb{P}^{N-1}$ is tangent to $X$ exactly along $\operatorname{Sing}(D)=\left\{y \in D \mid \operatorname{dim}\left(T_{y} D\right)>\operatorname{dim}(D)\right\}$.

We define the important notions of entry loci and $k$-secant defect and we study their first properties.

Definition 8.4 (Entry loci). Let $X \subset \mathbb{P}^{N}$ be a closed irreducible non-degenerate subvariety. Let us recall the diagram defining the higher secant varieties $S^{k} X$ as the join of $X$ with $S^{k-1} X$ :

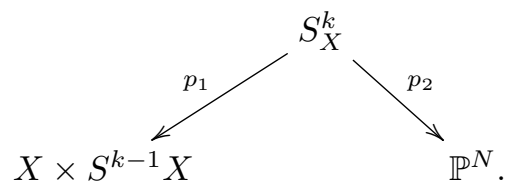

Let us define $\phi: X \times S^{k-1} X \rightarrow X$ to be the projection onto the first factor of this product.

Then, for $z \in S^{k} X$, the $k$-entry locus of $X$ with respect to $z$ is the scheme-theoretic image

$$
\Sigma_{z}^{k}=\Sigma_{z}^{k}(X):=\phi\left(p_{1}\left(p_{2}^{-1}(z)\right)\right)
$$


In the sequel mostly the 1-entry locus of a variety will be considered, which will simply be called the entry locus, and the suffix 1 will be omitted using the notation $\Sigma_{z}(X):=\Sigma_{z}^{1}(X)$. Sometimes we shall simply use $\Sigma_{z}$ without referring to $X$.

Geometrically, the support of $\Sigma_{z}^{k}$ is the locus described on $X$ by the $(k+1)$-secant $\mathbb{P}^{k}$ of $X$ passing through $z \in S^{k} X$. If $z \in S^{k} X$ is general, then through $z$ there passes an ordinary $(k+1)$-secant $\mathbb{P}^{k}$, i.e. given by $k+1$ distinct points on $X$. Thus for a general $z \in S^{k} X$ we can describe the support of $\Sigma_{z}^{k}$ in this way

$$
\left(\Sigma_{z}^{k}\right)_{\text {red }}=\overline{\left\{x \in X \mid \exists x_{1}, \ldots, x_{k} \text { distinct and } z \in\left\langle x, x_{1}, \ldots, x_{k}\right\rangle\right\}} \text {. }
$$

Moreover, by the Theorem on the dimension of the fibers of a morphism, for general $z \in S^{k} X$, the support of $\Sigma_{z}^{k}$ is equidimensional and every irreducible component contains ordinary $\mathbb{P}^{k}$ 's since necessarily $\operatorname{codim}(X)>k$ by the Trisecant Lemma. If $\operatorname{char}(K)=0$ and if $X$ is smooth, then for general $z \in S^{k} X$ the scheme $p_{1}^{-1}(z)$ is smooth so that $\Sigma_{z}^{k}$ is reduced.

To recover the scheme structure of $\Sigma_{z}^{k}$ geometrically, one could define $\Pi_{z}$ as the locus of $(k+1)$-secant $\mathbb{P}^{k}$ 's through $z$ and define $\Sigma_{z}^{k}=\Pi_{z} \cap X$ as schemes. For example, if through $z \in S X$ there passes a unique tangent line $L$ to $X$, then in this way we get $\Pi_{z}=L$ and $\Sigma_{z}=L \cap X$ consists of the point of tangency with the double structure taking into account the multiplicity of intersection.

Let us study the dimension of $\Sigma_{z}^{k}$ for $z \in S^{k} X$ general. First, let us remark that if $x \in \Sigma_{z}^{k}$ is a general point in an irreducible component, $z \in S^{k} X$ general, then, as sets,

$$
\phi^{-1}(x)=\left\{y \in S^{k-1} X \mid z \in\langle x, y\rangle\right\}=\langle z, x\rangle \cap S^{k-1} X \neq \emptyset
$$

and $\operatorname{dim}\left(\phi^{-1}(x)\right)=0$ because $z \in S^{k} X \backslash S^{k-1} X$ by the generality of $z$.

Definition 8.5 ( $k$-secant defect $\left.\delta_{k}\right)$. We define the $k$-secant defect of $X, 1 \leq k \leq k_{0}(X)$, $\delta_{k}(X)$, as the integer

$$
\delta_{k}(X)=\operatorname{dim}\left(\sum_{z}^{k}\right)=\operatorname{dim}\left(p_{1}\left(p_{2}^{-1}(z)\right)\right)=s_{k-1}(X)+\operatorname{dim}(X)+1-s_{k}(X),
$$

where $z \in S^{k} X$ is a general point.

For $k=1$, as advertised above we usually put $\Sigma_{z}=\Sigma_{z}^{1}$, for $z \in S X \backslash X$, and we define $\delta(X)=\delta_{1}(X)=2 \operatorname{dim}(X)+1-\operatorname{dim}(S X)$; for $k=0, \delta_{0}(X)=0$ by definition.

Sometimes we shall omit $X$ and simply use $\delta=\delta(X)$ to indicate the secant defect.

Let us reinterpret Terracini's Lemma from the point of view of these definitions.

Corollary 8.6 (Tangency along the entry loci). Let $X \subset \mathbb{P}^{N}$ be an irreducible closed subvariety. Let $k<k_{0}(X)$, i.e. $S^{k} X \subsetneq \mathbb{P}^{N}$, and let $z \in S^{k} X$ be a general point. Then:

1) the linear space $T_{z} S^{k} X$ is tangent to $X$ along $\left(\Sigma_{z}^{k}\right)_{\mathrm{red}} \backslash \operatorname{Sing}(X)$;

2) $\delta_{k}(X)<\operatorname{dim}(X)$;

3) $\delta_{k_{0}}(X)=\operatorname{dim}(X)$ if and only if $s_{k_{0}-1}(X)=N-1$, i.e. if and only if $S^{k_{0}-1} X$ is a hypersurface;

4) $s_{k}(X)=(k+1)(n+1)-1-\sum_{i=1}^{k} \delta_{i}(X)=\sum_{i=0}^{k}\left(\operatorname{dim}(X)-\delta_{i}(X)+1\right)$;

5) (cf. Corollary 7.3) if $X$ is a curve, $s_{k}(X)=2 k+1$, that is, $\delta_{k}(X)=0$ for every $k<k_{0}(X)$. 
REMARK 8.7. The statement of part 1) cannot be improved. Take for example a cone $X \subset \mathbb{P}^{5}$ of vertex a point $p \in \mathbb{P}^{5} \backslash \mathbb{P}^{4}$ over a smooth non-degenerate projective curve $C \subset \mathbb{P}^{4}$. If $z \in S(p, S C)=S X$ is general and if $z \in\langle x, y\rangle, x, y \in X$, it is not difficult to see that $\Sigma_{z}(X)=\langle p, x\rangle \cup\langle p, y\rangle$. The hyperplane $T_{z} S X$ is tangent to $X$ at $x$ and at $y$ by Terracini's Lemma so that it is tangent to $X$ along the rulings $\langle p, x\rangle$ and $\langle p, y\rangle$ minus the point $p$. Since $T_{p} X=\mathbb{P}^{5}$, the hyperplane $T_{z} S X$ is not tangent to $X$ at $p$ (neither $J$-tangent to $X$ at $p$ ).

REMARK 8.8. A phenomenon studied classically firstly by Scorza and then by Terracini is the case in which imposing tangency of a hyperplane at $k+1$ general points, $k \geq 0$, of a variety $X \subset \mathbb{P}^{N}$ forces tangency along a positive dimensional variety, even if $\delta_{k}(X)=0$.

Indeed, Terracini's Lemma says that if $\delta_{k}(X)>0, k<k_{0}(X)$, then a hyperplane tangent at $k+1$ points is tangent along the corresponding entry locus. The interesting and exceptional behavior occurs for varieties with $\delta_{k}(X)=0$. The first examples are the tangent developable to a non-degenerate curve or cones of arbitrary dimension. Indeed, they have $\delta_{0}=0$ as every variety but by imposing tangency at a general point, we get tangency along the ruling passing through the point.

Varieties for which a hyperplane tangent at $k+1, k \geq 0$, general points is tangent along a positive dimensional subvariety are called $k$-weakly defective varieties, according to Chiantini and Ciliberto [3]. We observe that for $k<k_{0}$ a $k$-defective variety, which for us means $\delta_{k}>0$, is $k$-weakly defective but the converse is not true, as recalled above.

In [3] many interesting properties of $k$-weakly defective varieties are investigated and a refined Terracini Lemma is proved, also by putting in modern terms the classification of $k$-weakly defective irreducible surfaces obtained classically by Scorza and Terracini. Let us remark that, as shown in [3], for every $k \geq 1$ there exist smooth varieties of dimension greater than one which are $k$-weakly defective but have $\delta_{k}(X)=0$ or $s_{k}(X)=$ $\min \{N,(k+1) n+k\}$.

As we have seen, the dual varieties encode information about the tangency of hyperplanes. Terracini's Lemma says that linear spaces containing tangent spaces to higher secant varieties are tangent along $\left(\Sigma_{z}^{k}\right)_{\text {red }} \backslash \operatorname{Sing}(X)$, see Corollary 8.6 Thus the maximal dimension of the fibers of $p_{2}: \mathscr{P}_{X} \rightarrow X^{*} \subset \mathbb{P}^{N^{*}}$ is an upper bound for $\delta_{k}(X)$ as soon as $S^{k} X \subsetneq \mathbb{P}^{N}$, as we shall immediately see. More refined versions with the higher Gauss maps $\gamma_{m}$, see below, can be formulated but in those cases the condition expressed by the numbers $\varepsilon_{m}(X)$, which can be defined as below, is harder to control.

THEOREM 8.9 (Dual variety and higher secant varieties). Let $X \subset \mathbb{P}^{N}$ be an irreducible non-degenerate projective variety. Let $p_{2}: \mathscr{P}_{X} \rightarrow X^{*} \subset \mathbb{P}^{N^{*}}$ be as above and let

$$
\varepsilon(X)=\max \left\{\operatorname{dim}\left(p_{2}^{-1}(H)\right), H \in X^{*}\right\} .
$$

If $S^{k} X \subsetneq \mathbb{P}^{N}$, then $\delta_{k}(X) \leq \varepsilon(X)$. In particular, if $p_{2}: \mathscr{P}_{X} \rightarrow X^{*}$ is a finite morphism, then $\operatorname{dim}\left(S^{k} X\right)=\min \{(k+1) n+k, N\}$.

Proof. Let $z \in S^{k} X$ be a general point. There exists an $x \in \Sigma_{z}^{k}(X) \cap X_{\text {reg }}$ and moreover $T_{z} S^{k} X$ is contained in a hyperplane $H$. Then

$$
p_{1}\left(p_{2}^{-1}(H)\right) \supseteq \operatorname{Sing}(X \cap H) \backslash(\operatorname{Sing}(X) \cap H),
$$


more precisely $\operatorname{Sing}(X \cap H) \backslash(\operatorname{Sing}(X) \cap H)$, contains the irreducible component of $\Sigma_{z}^{k}(X) \backslash$ $\left(\operatorname{Sing}(X) \cap \Sigma_{z}^{k}(X)\right)$ passing through $x$ by Corollary 8.6. Then $p_{1}\left(p_{2}^{-1}(H)\right)$ has dimension at least $\delta_{k}(X)=\operatorname{dim}\left(\Sigma_{z}^{k}(X)\right)$ and the conclusion follows.

For a smooth variety $X \subset \mathbb{P}^{N}$ the condition that $p_{2}: \mathscr{P}_{X} \rightarrow X^{*}$ is a finite morphism is equivalent to the ampleness of the locally free sheaf $\mathscr{N}_{X / \mathbb{P}^{N}}(-1)$, where $\mathscr{N}_{X / \mathbb{P}^{N}}$ is the normal bundle of $X$ in $\mathbb{P}^{N}$, see Exercise 9.8

COROllary 8.10 (cf. Corollaries 7.3 and 8.6. Let $X \subset \mathbb{P}^{N}$ be either an irreducible non-degenerate curve or a smooth non-degenerate complete intersection. Then

$$
\operatorname{dim}\left(S^{k} X\right)=\min \{(k+1) n+k, N\} .
$$

Proof. By Exercise 9.8 we know that for a smooth non-degenerate complete intersection $p_{2}: \mathscr{P}_{X} \rightarrow X^{*}$ is a finite morphism, a property which is immediate for projective curves. Thus the conclusions follow immediately from Theorem 8.9 .

9. Terracini's Lemma for $L G(4,8) \subset \mathbb{P}^{41}$ and integrability of non-degenerate symplectic Monge-Ampère equations in dimension four. It is well known that, for every $n \geq 3$, the secant variety $S \operatorname{LG}(n, 2 n) \subset \mathbb{P}^{N(n)}$ has the expected dimension $2 \operatorname{dim}(\operatorname{LG}(n, 2 n))+1$. By a clever use of Terracini's Lemma and by using the defining parametrization of $\mathrm{LG}(n, 2 n)$, Boralevi-Buczyński were able to compute the dimension of $S^{k} \mathrm{LG}(n, 2 n)$ for every $n \geq 4$ and for $k=2,3$. Their results were also independently discovered by Doubrov and Ferapontov in [6] although it is not completely clear if [6] treats the complex or the real case. We have the following results.

TheOREM 9.1 ([2], 6]). Let $\operatorname{LG}(n, 2 n) \subset \mathbb{P}^{N(n)}$ with $n \geq 4$. Thenrm:

1) $\operatorname{dim} S^{2} \mathrm{LG}(4,8)=31=(3 \times 10+2)-1$, i.e., $\delta_{2}(\mathrm{LG}(4,8))=1$;

2) $\operatorname{dim} S^{3} \mathrm{LG}(4,8)=39=(4 \times 10+3)-4$, i.e., $\delta_{3}(\mathrm{LG}(4,8))=4$;

$3)$ for $k=2,3$ the varieties $S^{k} \mathrm{LG}(n, 2 n) \subset \mathbb{P}^{N(n)}$ have the expected dimension for every $n \geq 5$.

By Terracini's Lemma and by the previous result, a hyperplane $H \subset \mathbb{P}^{41}$ such that

$$
H \supset T_{p} S^{2} \mathrm{LG}(4,8)
$$

is tangent to $\mathrm{LG}(4,8)$ along a curve, which is the entry locus $\Sigma_{p}^{2}(X)$ of $X$ with respect to $p \in S^{2} \mathrm{LG}(4,8)$ general.

A hyperplane $H \subset \mathbb{P}^{41}$ such that

$$
H \supset T_{q} S^{3} \mathrm{LG}(4,8)
$$

is tangent to $\operatorname{LG}(4,8)$ along a variety of dimension 4 , which is the entry locus $\Sigma_{q}^{3}(X)$ of $X$ with respect to $q \in S^{3} \mathrm{LG}(4,8)$ general. The condition $H \supset T_{q} S^{3} \mathrm{LG}(4,8), q \in$ $S^{3} \mathrm{LG}(4,8)$ general, means exactly

$$
[H] \in\left(S^{3} \mathrm{LG}(4,8)\right)_{\mathrm{reg}}^{*} .
$$

Now we can formulate the main result of [6].

Theorem 9.2 ([6]). A non-degenerate symplectic Monge-Ampère equation of dimension 4 corresponding to $[H] \in\left(\mathbb{P}^{41}\right)^{*}$ is integrable if and only if $[H] \in\left(S^{3} \mathrm{LG}(4,8)\right)_{\mathrm{reg}}^{*}$. 


\section{Exercises}

EXERCISE 9.3. Let $K$ be a(n algebraically closed) field of characteristic different from 2 and let $X=\nu_{2}\left(\mathbb{P}^{2}\right) \subset \mathbb{P}^{5}$ be the 2 -Veronese surface in $\mathbb{P}^{5}$. We shall identify $\mathbb{P}^{5}$ with

$$
\mathbb{P}\left(\left\{A \in M_{3 \times 3}\left((K) \mid A=A^{t}\right\}\right),\right.
$$

so that $X=\{[A] \mid \operatorname{rk}(A)=1\}$, as is well known. Prove the following facts:

(a) $S X=T X=V(\operatorname{det}(A)) \subset \mathbb{P}^{5}$ so that $S X$ is a cubic hypersurface and $\delta(X)=1$.

(b) If $x_{1}, x_{2} \in X$, then $T_{x_{1}} X \cap T_{x_{2}} X \neq \emptyset$ and, more precisely, it consists of a point for every $x_{1}, x_{2} \in X, x_{1} \neq x_{2}$.

(c) $\operatorname{Sing}(S X)=X$.

EXERCISE 9.4. Let $K$ be a(n algebraically closed) field and let $X=\mathbb{P}^{2} \times \mathbb{P}^{2} \subset \mathbb{P}^{8}$ be the Segre embedding of $\mathbb{P}^{2} \times \mathbb{P}^{2}$ in $\mathbb{P}^{8}$. Identifying $\mathbb{P}^{8}$ with

$$
\mathbb{P}\left(M_{3 \times 3}(K)\right),
$$

we have $X=\{[A] \mid \operatorname{rk}(A)=1\}$. Prove that:

(a) $S X=T X=V(\operatorname{det}(A)) \subset \mathbb{P}^{8}$ is the cubic hypersurface given by the cubic polynomial $\operatorname{det}(A)$;

(b) if $x_{1}, x_{2} \in X$, then $T_{x_{1}} X$ and $T_{x_{2}} X$ intersect in a line and $\delta(X)=2$;

(c) if $H$ is a general hyperplane in $\mathbb{P}^{8}$, then $Y:=X \cap H$ is a smooth, irreducible, non-degenerate 3 -fold $Y \subset \mathbb{P}^{7}$ such that $S Y=S X \cap H \subsetneq H$ so that $\delta(Y)=1$;

(d) given $y_{1}, y_{2} \in Y$, then $T_{y_{1}} Y \cap T_{y_{2}} Y \neq \emptyset$ (and it consists of a point if $y_{1}, y_{2} \in Y$ are general);

(e) $\operatorname{Sing}(S X)=X$.

(f) Let $p \in \mathbb{P}^{9} \backslash \mathbb{P}^{8}$, let $Z=S(p, X) \subset \mathbb{P}^{9}$ and let $X^{\prime}=Z \cap W$, with $W \subset \mathbb{P}^{9}$ a general hypersurface of degree $d \geq 2$ not passing through $p$. Then $X^{\prime}$ is a smooth, irreducible, non-degenerate 4-fold such that $S X^{\prime}=S Z=S(p, S X)$;

(g) Prove that $\operatorname{dim}\left(S X^{\prime}\right)=8$, yielding $\delta\left(X^{\prime}\right)=1$. Deduce from Terracini's Lemma that two general tangent spaces to $X^{\prime}$ intersect in a point, yielding $\delta\left(X^{\prime}\right)=1$.

Use the fact that $Z$ is a cone over $X$ to prove directly that two general tangent spaces to $X^{\prime}$ intersect in a point.

EXERCISE 9.5. Generalize part (f) of the previous problem and find the relation between $S X \subset \mathbb{P}^{N}$ and $S X^{\prime} \subset \mathbb{P}^{N+1}$ for $X^{\prime} \subset \mathbb{P}^{N+1}$ a general intersection of

$$
Z=S(p, X) \subset \mathbb{P}^{N+1}
$$

with a general hypersurface $W \subset \mathbb{P}^{N+1}$ of degree $d \geq 2$ not passing through the point $p \in \mathbb{P}^{N+1} \backslash \mathbb{P}^{N}$, which belongs to the vertex of $Z$.

EXERCISE 9.6. Let $X=\mathbb{P}^{m_{1}} \times \mathbb{P}^{m_{2}} \subset \mathbb{P}^{m_{1} m_{2}+m_{1}+m_{2}}$ be the Segre variety and let $p \in\left\langle x_{1}, x_{2}\right\rangle \in S X$ be a general point with $x_{i}=\left(a_{i}, b_{i}\right) \in X, x_{1} \neq x_{2}$ general. Show that the two-dimensional quadric $Q_{p}=\left\langle a_{1}, a_{2}\right\rangle \times\left\langle b_{1}, b_{2}\right\rangle$ is contained in $\Sigma_{p}$. Prove that $Q_{p}=\Sigma_{p}$ and deduce $\delta(X)=2$. 
ExERCISE 9.7. Prove the following facts, under the assumption char $(K)=0$ :

(a) If $X \subsetneq \mathbb{P}^{M} \subsetneq \mathbb{P}^{N}$ is a degenerate variety the dual variety $X^{*} \subset \mathbb{P}^{N *}$ is a cone of vertex $\left(\mathbb{P}^{M}\right)^{\perp}=\mathbb{P}^{N-M-1} \subset \mathbb{P}^{N *}$ over the dual variety of $X$ in $\mathbb{P}^{M}$.

(b) Suppose $X=S\left(L, X^{\prime}\right)$ is a cone of vertex $L=\mathbb{P}^{l}, l \geq 0$, over a variety $X^{\prime} \subset M=$ $\mathbb{P}^{N-l-1}, M \cap L=\emptyset$. Then $X^{*} \subset\left(\mathbb{P}^{l}\right)^{\perp}=\mathbb{P}^{N-l-1} \subset\left(\mathbb{P}^{N}\right)^{*}$ is degenerate. Is there any relation between $X^{*}$ and the dual of $X^{\prime}$ in $M$ ?

(c) Suppose $X \subset \mathbb{P}^{N}$ is a cone. Prove that $X^{*} \subset \mathbb{P}^{N *}$ is degenerate. Conclude that $X \subset \mathbb{P}^{N}$ is degenerate if and only if $X^{*} \subset \mathbb{P}^{N *}$ is a cone; and, dually, that $X \subset \mathbb{P}^{N}$ is a cone if and only if $X^{*} \subset \mathbb{P}^{N *}$ is degenerate.

(d) Assume now $p=\operatorname{char}(K)>0$ and show that for the irreducible curve $X \subset \mathbb{P}^{2}$ of equation $x_{0}^{p-1} x_{2}-x_{1}^{p}=0$ the dual curve is a line.

EXERCISE 9.8. Let $X \subset \mathbb{P}^{N}$ be a non-singular variety. Then, using Grothendieck's notation, prove that

$$
\mathscr{P}_{X} \simeq \mathbb{P}\left(N_{X / \mathbb{P}^{N}}(-1)\right)
$$

where $N_{X / \mathbb{P}^{N}}(-1)$ is the twist of the normal bundle of $X$ in $\mathbb{P}^{N}, N_{X / \mathbb{P}^{N}}$, by $\mathscr{O}_{\mathbb{P}^{N}}(-1)$ and that

$$
p_{2}: \mathscr{P}_{X} \rightarrow X^{*} \subset \mathbb{P}^{N *}
$$

is given by a sublinear system of $\left.\mid \mathscr{O}_{\mathbb{P}\left(N_{X / \mathbb{P}^{N}}(-1)\right)}(1)\right) \mid$.

(b) Deduce that for a smooth non-degenerate variety $X \subset \mathbb{P}^{N}$ the locally free sheaf $N_{X / \mathbb{P}^{N}}(-1)$ is ample, that is, $\mathscr{O}_{\mathbb{P}\left(N_{X / \mathbb{P}^{N}}(-1)\right)}(1)$ is ample, if and only if every $[H] \in X^{*}$ is tangent to $X$ in at most a finite number of points. In particular, for such varieties $\operatorname{def}(X)=0, \delta_{k}(X)=0$ for every $k<k_{0}(X)$ and a general tangent linear space $\mathbb{P}^{m}$, $m \geq \operatorname{dim}(X)$, is tangent to $X$ only at one point.

(c) Let $X^{n} \subset \mathbb{P}^{N}$ be a smooth non-degenerate complete intersection and let $c=$ $\operatorname{codim}(X)=N-\operatorname{dim}(X)$. If $I(X)=\left\langle f_{1}, \ldots, f_{c}\right\rangle$ with $f_{i}$ a homogeneous polynomial of degree $d_{i} \geq 2$, then

$$
N_{X / \mathbb{P}^{N}}(-1) \simeq \bigoplus_{i=1}^{c} \mathscr{O}_{X}\left(d_{i}-1\right)
$$

is an ample locally free sheaf. Deduce that $\operatorname{def}(X)=0$, that $\delta_{k}(X)=0$ for $k<k_{0}(X)$ and that a general tangent linear space $\mathbb{P}^{m}, m \geq \operatorname{dim}(X)$, is tangent to $X$ only at one point.

EXERCISE 9.9. Let $V$ be a complex vector space of dimension $N+1$ such that $\mathbb{P}(V)=\mathbb{P}^{N}$ and let $X \subset \mathbb{P}^{N}$ be a smooth non-degenerate variety of dimension $n \geq 1$.

Consider the restriction of the Euler sequence on $\mathbb{P}^{N}$ to $X$

$$
0 \rightarrow \Omega_{\mathbb{P}^{N} \mid X}^{1} \rightarrow V \otimes \mathscr{O}_{X}(-1) \rightarrow \mathscr{O}_{X} \rightarrow 0
$$

and the exact sequence on $X$

$$
0 \rightarrow N_{X / \mathbb{P}^{N}}^{*} \rightarrow \Omega_{\mathbb{P}^{N} \mid X}^{1} \rightarrow \Omega_{X}^{1} \rightarrow 0 .
$$

From these exact sequences define

$$
0 \rightarrow N_{X / \mathbb{P}^{N}}^{*}(1) \rightarrow V \otimes \mathscr{O}_{X} \rightarrow P_{X}^{1} \rightarrow 0,
$$


and

$$
0 \rightarrow \Omega_{X}^{1}(1) \rightarrow P_{X}^{1} \rightarrow \mathscr{O}_{X}(1) \rightarrow 0
$$

where $P_{X}^{1}$ is the so-called first jet bundle of $\mathscr{O}_{X}(1)$. Prove the following facts:

(a) For every closed point $x \in X$ we have $\mathbb{P}\left(P_{X}^{1} \otimes k(x)\right)=T_{x} X \subset \mathbb{P}(V)$.

(b) The surjection in 13 gives an embedding over $X$ of $\mathbb{P}\left(P_{X}\right) \rightarrow X$ into $\mathbb{P}(V) \times X \rightarrow$ $X$ in such a way that the restriction $\pi_{1}$ of the projection $\mathbb{P}^{N} \times X \rightarrow \mathbb{P}^{N}$ to $\mathbb{P}\left(P_{X}^{1}\right)$ maps $\mathbb{P}\left(P_{X}^{1}\right)$ onto $T X=\bigcup_{x \in X} T_{x} X$.

(c) Deduce from 13 that $\operatorname{det}\left(P_{X}^{1}\right) \simeq \operatorname{det}\left(N_{X / \mathbb{P}^{N}}(-1)\right) \simeq \omega_{X} \otimes \mathscr{O}_{X}(n+1)$ and use this result for $n=1$ to calculate/compare $\operatorname{deg}(T X)$ and $\operatorname{deg}\left(X^{*}\right)$.

ExERCISE 9.10. Let $C \subset \mathbb{P}^{N}, N \geq 2$, be an irreducible non-degenerate curve.

(a) Assume $\operatorname{char}(K)=0$ and prove that a general tangent linear space $\mathbb{P}^{m}$ with $m \geq 1$ is tangent to $C$ only at one point. Deduce in particular that a general tangent line to $C$ is tangent to $C$ only at one point.

(b) (Wallace, Kleiman) Let $p=\operatorname{char}(K)>0$, let $X \subset \mathbb{P}^{2}$ be the irreducible curve of equation $x_{0}^{p} x_{2}+x_{0} x_{2}^{p}-x_{1}^{p+1}=0$ and let $\left(u_{0}: u_{1}: u_{2}\right)$ be the dual coordinates on $\mathbb{P}^{2^{*}}$. Show that $X^{*} \subset \mathbb{P}^{2^{*}}$ has equation $u_{0}^{p} u_{2}+u_{0} u_{2}^{p}-u_{1}^{p+1}=0$ and that the Gauss map $\mathscr{G}_{X}: X \rightarrow \mathbb{P}^{2^{*}}$, which is naturally identified with $p_{2}: \mathscr{P}_{X} \rightarrow \mathbb{P}^{2^{*}}$, is bijective but not generically smooth. Deduce that $X^{* *}=X$ and that $X$ is not reflexive.

(c) Let $p=\operatorname{char}(K)>0$, let $N \geq 2$ and let $X \subset \mathbb{P}^{N}$ be the irreducible smooth hypersurface of equation

$$
\sum_{i=0}^{N} x_{i}^{p+1}=0 .
$$

Calculate $X^{*}$ and deduce that $X^{* *}=X$. Show directly that $X$ is not reflexive by observing that, if $p_{0}=\left(\alpha_{0}: \ldots: \alpha_{N}\right) \in X$, then the tangent hyperplane to $X$ at $q_{0}=\left(\alpha_{0}^{p}: \ldots: \alpha_{N}^{p}\right)=\mathscr{G}_{X}\left(p_{0}\right)$ is not tangent to $X$ at $p_{0}$.

(d) (Pardini) Let $p \geq 3$ be the characteristic of the base field $K$ and let $X \subset \mathbb{P}^{2}$ be the irreducible curve of equation $x_{0} x_{1}^{2 p}+x_{1} x_{2}^{2 p}+x_{2} x_{0}^{2 p}=0$. Show that $X^{*} \subset \mathbb{P}^{2^{*}}$ has degree $2(2 p+1)$ and that $X^{* *} \neq X$.

EXERCISE 9.11. Let $C \subset \mathbb{P}^{N}$ be a smooth non-degenerate projective curve of degree $d=\operatorname{deg}(X)$ and genus $g=g(C)$. Calculate geometrically as a function of $d$ and $g$ the following:

(a) $\operatorname{deg}(T C)$ for $N \geq 3$;

(b) $\operatorname{deg}(S C)$ for $N \geq 4$;

(c) $\operatorname{deg}\left(C^{*}\right)$ for $N \geq 2$.

EXERCISE 9.12. Prove the following facts.

(a) Let $X=\mathbb{P}^{1} \times \mathbb{P}^{n} \subset \mathbb{P}^{2 n+1}, n \geq 1$, be the Segre embedding of $\mathbb{P}^{1} \times \mathbb{P}^{n}$. Identify $\mathbb{P}^{2 n+1}$ with $\mathbb{P}\left(M_{2 \times(n+1)}(K)\right)$ and show that $\left(\mathbb{P}^{1} \times \mathbb{P}^{n}\right)^{*} \simeq \mathbb{P}^{1} \times \mathbb{P}^{n}$. Deduce that $\operatorname{def}\left(\left(\mathbb{P}^{1} \times \mathbb{P}^{n}\right)\right)=$ $n-1$.

(b) Show that if $X=\nu_{2}\left(\mathbb{P}^{2}\right) \subset \mathbb{P}^{5}$, or if $X=\mathbb{P}^{2} \times \mathbb{P}^{2} \subset \mathbb{P}^{8}$, then $X^{*} \simeq S X$. 


\section{References}

[1] B. Ådlandsvik, Joins and higher secant varieties, Math. Scand. 61 (1987), 213-222.

[2] A. Boralevi, J. Buczyński, Secants of Lagrangian Grassmannians, Ann. Mat. Pura Appl. 190 (2011), 725-739.

[3] L. Chiantini, C. Ciliberto, Weakly defective varieties, Trans. Amer. Math. Soc. 354 (2001), $151-178$.

[4] L. Chiantini, C. Ciliberto, On the concept of $k$-secant order of a variety, J. London Math. Soc. (2) 73 (2006), 436-454.

[5] J.-L. Clerc, Special prehomogeneous vector spaces associated to $F_{4}, E_{6}, E_{7}, E_{8}$ and simple Jordan algebras of rank 3, J. Algebra 264 (2003), 98-128.

[6] B. Doubrov, E. V. Ferapontov, On the integrability of symplectic Monge-Ampère equations, J. Geom. Phys. 60 (2010), 1604-1616.

[7] E. V. Ferapontov, L. Hadjikos, K. R. Khusnutdinova, Integrable equations of the dispersionless Hirota type and hypersurfaces in the Lagrangian Grassmannian, Int. Math. Res. Not. IMRN 2010, 496-535.

[8] G. Fischer, J. Piontkowski, Ruled Varieties - An Introduction to Algebraic Differential Geometry, Adv. Lectures Math., Vieweg, Braunschweig, 2001.

[9] L. Pirio, F. Russo, The XJC-correspondence, J. Reine Angew. Math. 716 (2016), 229-250.

[10] F. Russo, On the Geometry of Special Projective Varieties, Lect. Notes Unione Mat. Ital. 18, Springer, Cham, 2016.

[11] I. R. Shafarevich, Basic Algebraic Geometry, Grundlehren Math. Wiss. 213, Springer, Berlin, 1974.

[12] A. Terracini, Sulle $V_{k}$ per cui la varieta' degli $S_{h}(h+1)$-secanti ha dimensione minore dell' ordinario, Rend. Circ. Mat. Palermo 31 (1911), 392-396.

[13] A. Terracini, Alcune questioni sugli spazi tangenti e osculatori ad una varietá, I, II, III, in: Selecta Alessandro Terracini, vol. I, 24-90, Edizioni Cremonese, Roma, 1968. 